NASA/TM-2005-213595

\title{
Strain Rate Sensitivity of Epoxy Resin in Tensile and Shear Loading
}

\author{
Amos Gilat \\ Ohio State University, Columbus, Ohio \\ Robert K. Goldberg and Gary D. Roberts \\ Glenn Research Center, Cleveland, Ohio
}


Since its founding, NASA has been dedicated to the advancement of aeronautics and space science. The NASA Scientific and Technical Information (STI) Program Office plays a key part in helping NASA maintain this important role.

The NASA STI Program Office is operated by Langley Research Center, the Lead Center for NASA's scientific and technical information. The NASA STI Program Office provides access to the NASA STI Database, the largest collection of aeronautical and space science STI in the world. The Program Office is also NASA's institutional mechanism for disseminating the results of its research and development activities. These results are published by NASA in the NASA STI Report Series, which includes the following report types:

- TECHNICAL PUBLICATION. Reports of completed research or a major significant phase of research that present the results of NASA programs and include extensive data or theoretical analysis. Includes compilations of significant scientific and technical data and information deemed to be of continuing reference value. NASA's counterpart of peerreviewed formal professional papers but has less stringent limitations on manuscript length and extent of graphic presentations.

- TECHNICAL MEMORANDUM. Scientific and technical findings that are preliminary or of specialized interest, e.g., quick release reports, working papers, and bibliographies that contain minimal annotation. Does not contain extensive analysis.

- CONTRACTOR REPORT. Scientific and technical findings by NASA-sponsored contractors and grantees.
- CONFERENCE PUBLICATION. Collected papers from scientific and technical conferences, symposia, seminars, or other meetings sponsored or cosponsored by NASA.

- SPECIAL PUBLICATION. Scientific, technical, or historical information from NASA programs, projects, and missions, often concerned with subjects having substantial public interest.

- TECHNICAL TRANSLATION. Englishlanguage translations of foreign scientific and technical material pertinent to NASA's mission.

Specialized services that complement the STI Program Office's diverse offerings include creating custom thesauri, building customized databases, organizing and publishing research results ... even providing videos.

For more information about the NASA STI Program Office, see the following:

- Access the NASA STI Program Home Page at http://www.sti.nasa.gov

- E-mail your question via the Internet to help@sti.nasa.gov

- Fax your question to the NASA Access Help Desk at 301-621-0134

- Telephone the NASA Access Help Desk at 301-621-0390

- Write to:

NASA Access Help Desk

NASA Center for AeroSpace Information 7121 Standard Drive

Hanover, MD 21076 
NASA/TM-2005-213595

\title{
Strain Rate Sensitivity of Epoxy Resin in Tensile and Shear Loading
}

\author{
Amos Gilat \\ Ohio State University, Columbus, Ohio \\ Robert K. Goldberg and Gary D. Roberts \\ Glenn Research Center, Cleveland, Ohio
}

National Aeronautics and

Space Administration

Glenn Research Center 
Trade names or manufacturers' names are used in this report for identification only. This usage does not constitute an official endorsement, either expressed or implied, by the National Aeronautics and Space Administration.

Available from

NASA Center for Aerospace Information 7121 Standard Drive

Hanover, MD 21076
National Technical Information Service 5285 Port Royal Road Springfield, VA 22100

Available electronically at http:/ /gltrs.grc.nasa.gov 


\title{
Strain Rate Sensitivity of Epoxy Resin in Tensile and Shear Loading
}

\author{
Amos Gilat \\ Ohio State University \\ Department of Mechanical Engineering \\ Columbus, Ohio 43210 \\ Robert K. Goldberg and Gary D. Roberts \\ National Aeronautics and Space Administration \\ Glenn Research Center \\ Cleveland, Ohio 44135
}

\begin{abstract}
The mechanical response of E-862 and PR-520 resins is investigated in tensile and shear loadings. At both types of loading the resins are tested at strain rates of about $5 \times 10^{-5}, 2$, and 450 to $700 \mathrm{~s}^{-1}$. In addition, dynamic shear modulus tests are carried out at various frequencies and temperatures, and tensile stress relaxation tests are conducted at room temperature. The results show that the toughened PR-520 resin can carry higher stresses than the untoughened E-862 resin. Strain rate has a significant effect on the response of both resins. In shear both resins show a ductile response with maximum stress that is increasing with strain rate. In tension a ductile response is observed at low strain rate $\left(\sim 5 \times 10^{-5} \mathrm{~s}^{-1}\right)$, and brittle response is observed at the medium and high strain rates $\left(2\right.$, and $\left.700 \mathrm{~s}^{-1}\right)$. The hydrostatic component of the stress in the tensile tests causes premature failure in the E-862 resin. Localized deformation develops in the PR-520 resin when loaded in shear. An internal state variable constitutive model is proposed for modeling the response of the resins. The model includes a state variable that accounts for the effect of the hydrostatic component of the stress on the deformation.
\end{abstract}

\section{Introduction}

Resins are frequently used as the matrix material in laminated fibrous composites (glass or carbon fibers in resin matrix) that are used in applications where loading is applied at different rates. The mechanical properties (stress-strain relation and failure) of composites, like other materials, are sensitive to the rate at which they are loaded and deformed. The rate dependency of these properties is determined from tests in which material specimens are loaded at different strain rates.

In many engineering applications the strain rate at which materials are loaded ranges from $10^{-5} \mathrm{~s}^{-1}$ in quasi-static loading to $10^{3} \mathrm{~s}^{-1}$ in dynamic loading. Within this range hydraulic testing machines are typically used for testing at strain rates up to about $5 \mathrm{~s}^{-1}$, and the Split Hopkinson Bar (SHB) technique is used for testing at strain rates above about $200 \mathrm{~s}^{-1}$. In the split Hopkinson bar technique a short material specimen is placed between two bars. The specimen is loaded by a wave that is generated in one of the bars (more details about the technique are given in the next section). The technique was introduced by Kolsky (1949) with loading in compression and has been later modified to torsion and tensile loading.

With metals, most of the low and medium strain rate tests are done in tension. In studies that include high strain rate tests with the SHB technique, most of the tests are done in compression since compression SHB tests are the easiest to conduct. A large number of tests have also been conducted in shear (torsion of a thin-walled tube) but much fewer tests have been done in tension. This is because torsion and especially tensile SHB tests are more difficult to conduct than the compression tests. For metals, testing with different types of loading, which require specimens with different geometries, does not present a significant problem since metals are homogeneous and isotropic, and specimens can easily be machined 
to have any geometry. The results show consistent material behavior that is independent of the type of loading. This is because at room temperature over a wide range of strain rates, the strain rate sensitivity in metals is mostly due to the time dependency of dislocations motion that can be modeled in terms of various kinds of applied loads.

With laminated fibrous composites, investigating the effects of strain rate on the material response is by far more complicated. A unidirectional lamina, which is not homogeneous and not isotropic, is the basic building block of a multi-layered laminate. Within the laminate, the stress, strain, and strain rate are not uniform and vary from ply to ply. With laminated composites it is also more difficult to conduct, and correlate between, experiments with different types of loadings. A thin laminate is suitable for tensile loading, but a thicker laminate is required for compression loading. Pure shear properties of a lamina are difficult (or impossible) to measure directly, while laminas that are part of a multi-ply composite can undergo significant deformation in shear. In addition, the strain rate sensitivity of the composite is due to the rate sensitivity of the fibers and the resin matrix. The relationship between load and deformation in resins is also more complicated than in metals since the hydrostatic component of the stress has a significant effect even at low level of stress.

Tensile and compression tests with different configurations of laminates at various strain rates show that the response of composites is greatly affected by strain rate. In general, experimental results show an increase of modulus and strength with increasing strain rate. The development of constitutive equations that include the effect of strain rate from the results of tests with laminates alone is not straightforward. This is because the actual stress and deformation in the matrix and the fibers depends on the configuration of the laminate and, as mentioned before, within the laminate varies from ply to ply. For example, in a $\pm \theta$ angle-ply laminate that is loaded in uniaxial tension, the stress in the matrix can be a combination of compression and shear for small $\theta$ 's, or of tension and shear for large $\theta$ 's. Obviously, the behavior of the resin under different types of loads and loading rates has to be understood before a general model that includes strain rate effects can be developed for the composite.

Only limited data is available on the strain rate sensitivity of polymers and resins, and most of the data that includes high strain rates is from compression tests. Results from such tests, Walley et al. (1989), Chen and Zhou (1988), Shah Khan et al. (2002), Buckley et al. (2001), show an increase in yield stress and maximum stress with increasing strain rate. Buckley et al. (2001) have also conducted tensile tests, which showed an increase in modulus, a small increase in maximum stress, and a reduction in failure strain with increasing strain rate. In addition, the resins show a much more brittle response in tension than in compression. The same resins that were tested by Buckley et al. (2001) were also tested in shear at various strain rates by Hou et al. (2000). Their results show a more ductile response in shear than what was observed in tension. Tests with various types of combined loading ranging from uniaxial compression to biaxial tension were carried out by Kody and Lesser (1997). These tests were done at different strain rates within the range of a hydraulic machine. The results show that in addition to strain rate, the hydrostatic component of the stress has a very significant effect on the yield stress.

Polymers are known to have a strain rate dependent deformation response that is nonlinear. Traditionally, viscoelasticity models have been used to capture this behavior (Wineman and Rajagopal (2000)). However, there has been an interest in the research community in using constitutive equations developed for metals, based on viscoplasticity theory, to model the nonlinear, strain rate dependent deformation of polymers. For example, Zhang and Moore (1997) adapted the Bodner-Partom internal state variable based viscoplasticity model (Bodner 2002) developed for metals to analyze the nonlinear, uniaxial tensile response of polyethylene. Bordonaro (1995) and Krempl and Ho (2000) adapted the viscoplasticity theory based on overstress (originally developed for metals) to analyze the nonlinear deformation of Nylon 66.

Unlike in metals, hydrostatic stresses are known to affect the yield stress and nonlinear response of polymers (Ward (1983)). For example, the absolute value of the yield stress in compression is higher than the yield stress in tension. To account for these effects within a viscoplasticity approach, researchers such as Pan and colleagues (Li and Pan (1990), Chang and Pan (1997)) and Hsu et al. (1999) incorporated the effects of hydrostatic stresses into their models by applying variations of the Drucker-Prager yield criteria 
(Khan and Huang (1995)). In these equations, the definitions of the effective stress and effective inelastic strain rate are modified to account for the effects of hydrostatic stresses. However, while efforts to account for the effects of hydrostatic stresses in developing yield criteria for polymers have been reasonably successful, the constitutive equations developed to model the full range of the nonlinear deformation response have not been fully successful.

The objective of the research presented here is to study the response of two types of epoxy resins, that are frequently used for fabrication of aerospace composites by resin transfer molding, in tensile and shear loading over a wide range of strain rates. E-862 is an untoughened two-part epoxy system (EPON Resin 862/EPON Curing Agent W) made by Shell Chemical, and PR-520 is a one-part toughened epoxy resin made by Cytec. The resins were loaded in shear and in tension in constant strain rate tests over a wide range of strain rates. The shear and tension loadings provide an opportunity to examine the effect of the hydrostatic stress component on the material response. In addition, dynamic shear modulus tests were conducted at various frequencies and several temperatures, and stress relaxation tests were conducted at room temperature. An internal state variable constitutive model is used to simulate the experiments. The model is based on the constitutive equations of Bodner (2002), which is modified to include the effect of the hydrostatic component of the stress on the response. This modification is done by introducing a new internal state variable.

\section{Experimental Setup}

Tension and torsion tests have been conducted at strain rates of approximately $5 \times 10^{-5}, 2$, and 400 to $700 \mathrm{~s}^{-1}$. The low and medium strain rate tests $\left(5 \times 10^{-5}\right.$ and $\left.2 \mathrm{~s}^{-1}\right)$ are done using a hydraulic bi-axial (tension/torsion) Instron machine, and the high strain rate tests are done using a tensile or a torsional Split Hopkinson Bar (SHB) apparatus. Dynamic measurements of shear modulus were performed over a range of temperatures and frequencies using the ARES instrument made by TA Instruments. The measurements were done using torsion rectangular specimens and a dynamic strain amplitude of 0.002 . Temperatures ranged from room temperature to $250{ }^{\circ} \mathrm{C}$, and frequencies ranged from 0.1 to $100 \mathrm{rad} / \mathrm{s}(0.0159$ to $15.9 \mathrm{~Hz}$ ), which correspond to strain rate range of $2 \times 10^{-4}$ to $0.2 \mathrm{~s}^{-1}$. Stress relaxation tests were done using the DMA 2980 Dynamic Mechanical Analyzer made by TA Instruments. These measurements were made at $30{ }^{\circ} \mathrm{C}$ using single cantilever beam specimens and a strain of 0.01 .

The split Hopkinson bar apparatus, shown schematically in figure 1, is made up of two aluminum bars. The specimen is placed (cemented) between the bars. The specimen is loaded by a wave that is generated in the incident bar by first clamping a tensile force in the tensile SHB configuration, or a torque in the torsional SHB configuration, in the end section of the incident bar, and then releasing the clamp. Upon loading, part of the loading wave reflects back to the incident bar and part propagates on through the specimen to the transmitter bar. The incident and transmitter bars remain elastic throughout the test. The waves in the bars are measured with strain gages at two locations on the incident bar, and one location on the transmitter bar (Gage A, Gage B, and Gage C in fig. 1). The strain rate, strain, and stress in the specimen during the test are determined from the recorded waves in the bars. The details of the SHB technique are described in Staab and Gilat (1995) for the tension tests, and Gilat (2000) for the torsion tests.

\section{Specimens}

\section{Tensile Tests}

Three types of specimens are used in the tensile tests. All of the tests in the Instron machine and many of SHB tests are done with specimens having one of two kinds of dog-bone geometry, shown in figures 2(a) and 2(b). The specimen in figure 2(a) has a uniform thickness of $7.1 \mathrm{~mm}$, while in figure 2(b) the 
thickness of the specimen in the gage section is reduced to $2.8 \mathrm{~mm}$. A third type of specimen, which is a very short cylindrical specimen, as shown in figure 3, is used in some of the tests with the split Hopkinson bar apparatus. The specific situations in which the different types of specimens are used will be described later in this article. The specimen coupons are glued into two cylindrical aluminum adapters. For use in the split Hopkinson bar the unit (a specimen glued to two adapters) is cemented between the incident and transmitter bars. For use in the hydraulic testing machine the unit is pinned to double universal joints, which are connected to the grips of the machine. The double universal joint connection reduces the possibility of introducing a bending moment resulting from a possible eccentric load line in the testing machine.

\section{Torsion Tests}

In the torsion tests the specimen is a short thin-walled tube, shown in figure 4 . The specimen is obtained by machining a notch in a thick-walled tube. The thick-walled tube is made from an epoxy plate such that the axis of the tube is perpendicular to the plate. The specimen is first glued to aluminum adapters, and the unit is then attached to the testing machine. In a torsional split Hopkinson bar test the unit (a specimen glued to two adapters) is cemented between the incident and transmitter bars. In a test with the Instron machine, the unit is attached mechanically to the machine. The adapters in this case have a hexagonal end that is attached by screws to a hexagonal grip. To examine the effect of thickness of the specimen on the results, specimens with a wall thickness of $0.63 \mathrm{~mm}$ and $1.27 \mathrm{~mm}$ are used.

\section{Modulus and Relaxation Tests}

Specimens for the dynamic shear modulus tests and stress relaxation tests were cut from the same molded sheets used to make specimens for the tensile and torsion test specimens described above. Specimens were approximately $7.3 \mathrm{~mm}$ wide and $2.5 \mathrm{~mm}$ thick. The gage lengths of the torsion rectangular specimens were approximately $24 \mathrm{~mm}$, and the beam lengths of the single cantilever beam specimens were approximately $17 \mathrm{~mm}$.

\section{Measurement of Stress and Strain}

\section{Tests with the Instron Machine}

In tests with the Instron machine the load cell of the machine measures the force, or the torque, applied to the specimens. The tensile, or shear, stress is determined from the force, or torque, by assuming a uniform state of uniaxial tension or pure shear in the specimen gage length. The strain in the tensile tests is measured with strain gages that are cemented to the specimen. Two strain gages (Measurements Group EA-06-125BZ-350 or EA-06-031DE-350) are cemented on the specimen's surface on opposite sides. The strain can also be determined from the displacement of the machine's head. In the torsion tests the shear strain is determined from the relative rotation between the specimen's ends. The relative rotation is measured by the rotation of the machine's head and by a special device made of two rings that are attached at the ends of the specimen gage and an LVDT that measure the relative rotation between the rings, Gilat and Krishna (1977).

\section{Tests with the SHB Technique}

In the standard SHB technique, the history of stress and strain in the specimen is determined from the recorded elastic waves in the bars. In this determination it is assumed that the specimen is under a state of uniform uniaxial tension stress and deformation in the tensile test and pure shear in the torsion test. As described later, in many of the tensile split Hopkinson bar tests the strain was also measured directly on 
the specimen. This is done by placing two strain gages on opposite sides of the specimen just as in the tests with the Instron machine.

\section{Modulus and Relaxation Tests}

In the dynamic shear modulus tests a sinusoidal strain with amplitude $\gamma$ is applied to a torsion rectangular specimen, and the resulting torque is measured. The shear stress $\tau$ is calculated from the torque using standard equations for torsion of rectangular beams (Rheometric Scientific, Inc, Instrument Manual 902-30026, Rev A, pages 54, 59, June 2000.) The stress, which lags the applied strain by a phase angle $\delta$, can be resolved into sinusoidal components that are in phase and out of phase with the applied strain. The in-phase component is the elastic response, and the out-of-phase component is the viscous response. The in-phase (elastic) modulus, $G^{\prime}$, is called the "storage modulus", and is defined by

$$
G^{\prime}=(\tau / \gamma) \cos \delta
$$

[Note that for a perfectly elastic material the torque and strain are completely in phase (i.e. $\delta=0$, $\cos \delta=1$ ), and the equation above becomes the standard equation for elastic shear modulus.] The out-ofphase modulus, $G$ ", is called the "loss modulus" and is defined by

$$
G^{\prime \prime}=(\tau / \gamma) \sin \delta
$$

In the stress relaxation tests a constant strain, $\gamma$, is applied to a single cantilever beam, and the decaying force, $f(t)$, is measured. The time-dependent tensile stress, $\sigma(t)$, is calculated from the force using standard beam bending equations. (TA Instruments, Inc., DMA 2980 Dynamic Mechanical Analyzer Operator's Manual, PN 984004.002, Rev J, March 2002.)

There are some potential sources of inaccuracy in the dynamic shear modulus and the stress relaxation tests. The equations in the instrument manuals for calculating tensile and shear moduli are assumed to be valid, and the default value of 0.44 is used for the Poisson's ratio in the beam bending equations. Since the purpose for performing the dynamic mechanical tests is to quickly determine the relative effects of strain rate and temperature on the material modulus, no effort was made to measure the actual Poisson's ratio or to verify the accuracy of the stresses calculated using the beam bending and torsion equations.

\section{Experimental Results}

Tensile and torsion tests to failure have been conducted with specimens made of E-862 and PR-520 epoxy resins at strain rates of approximately $5 \times 10^{-5}, 2$, and 400 to $700 \mathrm{~s}^{-1}$. Examples of data recorded and processed in typical experiments are shown in figures 5 to 10. Figure 5 shows the stress and strain as a function of time in a low rate torsion test. The figure shows that the shear strain determined from the machine's head rotation and from the relative-rotation-measuring device are almost the same. This is because the connection of the specimen to the machine and the machine itself are very stiff relative to the specimen. Data recorded in a torsional SHB test is shown in figure 6. The figure shows the waves that are recorded in the incident and transmitted bars at the three gage locations shown in figure 1 . The stress, strain rate, and strain determined from these waves by using the SHB equations are shown in figure 7. The figure shows that following the rise time the strain rate in the specimen is not constant but is decreasing gradually. This is because the strain rate in the SHB test depends on the response of the specimen and as the specimen's stress increases, the strain rate decreases. Figure 8 shows records from a 
low strain rate tensile test in the Instron machine. The figure shows the stress, determined from the measured force, the strain, determined from the head displacement, and the strain measured by the two strain gages that are attached to the specimen, all as a function of time. The figure shows that the strain measured by the two strain gages is almost identical which means that there is no bending. The strain determined from the head displacement is larger than the strain from the strain gages. This is because in determining the strain from the head displacement it is assumed that all the displacement is due to deformation in the gage section of the specimen. In reality, however, the displacement of the head is also due to deformation in the fillets (the rounded ends) and deformation in the other components that are loaded (adapters, connecting pins, universal joints). Figure 9 shows records from a tensile SHB test with a dog-bone shaped specimen. The figure shows the elastic waves recorded in the bars and the strain measured by the strain gages that are attached to the specimen. The stress, strain rate, and strain that are determined from these waves are shown in figure 10. The strain from the specimen's strain gages is smaller than the strain determined from the elastic waves. This is again due to the fact that in the calculations using the elastic waves, it is assumed that all the deformation is taking place in the gage section.

\section{Results for E-862 Resin}

The results for the E-862 resin are shown in figures 11 to 16 . The shear stress-strain curves from all the torsion tests are shown together in figure 11. The following observations can be made from this figure:

- The maximum stress is very sensitive to the strain rate. The maximum stress increases with increasing strain rate. In the high strain rate tests the maximum stress is about twice the maximum stress in the low strain rate tests.

- The E-862 resin is relatively ductile in shear. The maximum strain, however, decreases with strain rate. In the low strain rate tests the maximum strain reaches 0.4. At high rate the maximum strain is about half of that.

- The results are independent of the wall-thickness of the specimen. Results from tests with wall thicknesses of about 0.63 and $1.27 \mathrm{~mm}$ are indistinguishable.

Figure 12 shows the tensile stress-strain curves from all the tensile tests. The following observations can be made from this figure:

- The maximum stress is about the same in the intermediate and high strain rates tests and lower in the low rate tests.

- The material response in tension is much more brittle than in shear. In the low strain rate test, however, the response is much more ductile than in the medium and high strain rate tests.

The tensile tests were initially done with the dog-bone shaped specimens, shown in figure 2(a). It was observed that, in tests at moderate and high strain rates, the specimens fractured in the fillet, as shown in figure 13(a). It appears that the specimens fractured in the fillet and not in the gage section that has the smaller cross-sectional area because of the high tensile hydrostatic stress component that exists in the fillet due to the curvature of the edges. Subsequently, the geometry of the specimen was changed to the one shown in figure 2(b). This specimen is the same as the original one except that the thickness perpendicular to the plane of the page in the gage section is reduced to $2.8 \mathrm{~mm}$. (The specimen has a dogbone shape in two directions.) The objective was to increase the stress in the gage section such that the specimen will fail in the gage section and not in the rounded ends. In addition, some of the tests were done also by using smaller strain gages (Measurements Group EA06-031 DE-350) for measuring the 
strain. In tests with reduced area specimens that have strain gages, the specimens fractured at the edge of the gage as shown in figure 13(b). To examine the effect of the strain gages, additional tests were conducted without strain gages cemented to the specimen. In these tests the strain that was determined from the stroke of the Instron machine, or from the SHB equations, was corrected empirically according to the relationship between this strain and the strain measured by the strain gages in experiments where they were used. In tests where no strain gages are used, the maximum stress and strain are the highest and the specimens fracture in the gage section. The differences between the different types of tests are shown in figure 14 for the tests at the medium strain rates. This figure shows that while the slope of the stressstrain curve is about the same in all the tests, the maximum stress is the lowest in the specimens with the standard cross-sectional area and large strain gages, and largest in the specimens with the reduced crosssectional area and no strain gages. The maximum stress in tests with reduced area and strain gages is in between, with higher stress in the experiment with the smaller strain gage.

Most of the tensile SHB tests were done with the dog-bone shaped specimens (fig. 2) that were used in the low and medium rate tests. The intention was to eliminate a possible effect of the specimen geometry on the results. The dog-bone shaped specimen that is shown in figure 2 is, however, relatively long for the SHB test. Ideally in the Split Hopkinson bar experiment, the specimen is short such that the time for the stress waves to traverse the gage section is short and the specimen is in a homogeneous state of stress. With a relatively long specimen, the stress-strain curves that are obtained from the tests contain oscillations that are due to the back and forth waves in the specimen, as can be seen in figure 12 . To comply with the requirements of the SHB technique, experiments were also done with short cylindrical specimens, figure 3 . The stress-strain curves from these tests have no oscillations and are in agreement with the results from tests with the longer specimens. The differences between the results from the various tensile SHB tests are shown in figure 15. In the first three tests with the standard cross-sectional area and the larger strain gages, the maximum stress is about $40 \mathrm{MPa}$ (plot (a)). When the cross sectional area is reduced, the max stress is about the same when the large strain gages are used, but the stress reaches about $75 \mathrm{MPa}$ when small strain gages are used (plot (b)). Results from tests with specimens with reduced area and without strain gages shows much higher strains (plot (c)). The stress-strain curve, however, has large oscillations due to the long length of the specimen. In the tests with the short cylindrical specimens (plot (d)) there are no oscillations. In one test the stress reached about $85 \mathrm{MPa}$, which is the highest stress level from all the tests. The failure of two of the short cylindrical specimens was, however, affected by hydrostatic tension. As shown in figure 16, these specimens fractured outside the gage section in the region that is actually inside the aluminum adapter.

Overall, the results from using specimens with different geometries show that at the moderate strain rate, with reduced cross-sectional area (to limit hydrostatic stress effects) and no strain gages (to remove stress concentrations due to the gage and/or its glue), there is a noticeable increase in max stress over the low strain rate tests. In the high rate tests, on the other hand, making all the corrections improved the maximum stress somewhat, but there is still most likely premature failure, since it occurs outside the gage section. In reality the failure stress at the high strain rate is most likely higher than in the moderate strain rate.

\section{Results for PR-520 Resin}

The results for PR-520 are shown in figures 17 to 20 . Figure 17 shows the measured shear stressstrain curves from all the torsion tests. The following observations can be made from the figure:

- The maximum stress is very sensitive to the strain rate. The maximum stress increases with increasing strain rate. For the high strain rate tests, the maximum stress is nearly twice the maximum stress in the low strain rate tests.

- The PR-520 resin is relatively ductile. Unlike the E-862, the ductility is independent of the strain rates. At all of the strain rates the strain reached at least 0.4 . 
- At all strain rates the stress reaches a maximum at strain of about 0.15 . Then there is a reduction of stress with increasing strain. The reduction is larger in the medium and high strain rate tests than in the low strain rate tests. The stress reduction might be due to localized deformation that develops in these tests.

- The results are independent of the wall-thickness of the specimen. Results from tests with wall thickness of about 0.025 and 0.049 in. are indistinguishable.

Figure 18 shows tensile stress-strain curves from all the tests. The following observations can be made from the figure:

- The maximum stress is about the same in the intermediate and high strain rates and lower in the low rate test.

- The material response in tension is brittle compared with the response in shear. In the low strain rate, however, the response is much more ductile than in the medium and high strain rate tests.

Unlike the E-862 resin, the failure of the PR-520 resin does not appear to be sensitive to the hydrostatic component of the stress. None of the PR-520 resin specimens fractured in the rounded fillet region. The presence of strain gages on the specimen has only a minor effect on the failure in the medium and high strain rate tests. For the medium strain rate tests, the results from tests with different types of specimens are shown in figure 19. The figure shows that the stress strain curves are very similar except for a small difference in the maximum stress. The maximum stress is lowest in specimens with the standard area and large strain gages, and largest in specimens with the reduced area and no strain gages. For the SHB tests the results from tests with different specimens are shown in figure 20. As with the E-862 resin, the stress strain curves from tests with the long dog-bone shaped specimen have oscillations and the curves from the tests with the short cylindrical specimens are smooth. No methodical differences are observed with regard to the maximum stress, except that the maximum stress from one of the tests with a long specimen and without strain gages attached is higher than the rest.

\section{Results of Modulus and Relaxation Tests}

The variation in dynamic shear modulus during a constant rate temperature ramp is one method used to determine the glass transition temperature, $T_{g}$, of polymer resins. Data of this type is shown in figures 21 and 22 for measurements at a frequency of $10 \mathrm{rad} / \mathrm{s}(1.59 \mathrm{~Hz})$. One method for calculating $T_{g}$ from these curves is to determine the intercept of the linear regions above and below the glass transition, as shown in figures 21 and 22. Using this approach, $T_{g}=133^{\circ} \mathrm{C}$ for the E-862 resin, and $T_{g}=148{ }^{\circ} \mathrm{C}$ for the PR-520 resin. The allowable service temperature for structural composites is typically at least $25^{\circ}$ (and often $50{ }^{\circ} \mathrm{C}$ ) below $T_{g}$. In this temperature range the resin is mainly elastic in character, as indicated by the large value of $G^{\prime}$ compared to $G^{\prime \prime}$. In this elastic (or "glassy") region, the modulus decreases with increasing temperature primarily because thermal expansion leads to an increased molecular spacing, which reduces intermolecular forces. Although the viscous component is small, the viscoelastic nature of the material causes the modulus to be rate and time dependent. The rate dependence at several temperatures is shown in figures 23 and 24 . The slight increase in modulus with increasing frequency is typical for polymers in the glassy region. The time dependence is shown in the stress relaxation curves at $30^{\circ} \mathrm{C}$ in figures 25 and 26 . The stress decreases by about 10 percent after 60 minutes. The shear modulus measurements at temperatures above $T_{g}$ also provide useful information about the behavior of the resin. In figures 21 and 22, $G^{\prime}$ decreases sharply and then levels off in a plateau region as the temperature is increased beyond $T_{g}$. In the plateau region, thermal energy is high enough to overcome barriers to local motion of the polymer chain segments, and the stiffness is determined by the permanent crosslinked network of the polymer chains. The magnitude of $G^{\prime}$ in the plateau region is an indication of the crosslink density. Resins that are highly crosslinked have a higher value of $G^{\prime}$ in the plateau region and tend to be more brittle. The higher value of $G^{\prime}$ in the plateau region for E-862 resin compared to PR-520 
resin suggests a higher crosslink density and is consistent with the observation of more brittle behavior for the E-862 resin.

\section{Discussion of the Experimental Results}

The response of the E-862 and PR-520 resins is strongly affected by the rate of deformation and the type of loading. In shear both resins are ductile with a maximum strain that exceeds 0.2 . The ductility of the E-862 resin decreases with increasing strain rate and the PR-520 resin shows evidence of localized deformation. The strain rate has a very significant effect on the maximum shear stress, which increases considerably with increasing strain rate. In tension both resins are much more brittle. At the intermediate and high strain rates the response is essentially linear to failure. At the low strain rate an initial linear response is followed by a non-linear behavior. Near the end, the slope of the stress-strain curves of the E-862 resin is very small. For the PR-520, the curves end with a plateau or even a negative slope. The hydrostatic component of the stress appears to affect the response in tension. This is especially evident in the E-862 resin where the specimens often fractured outside the gage section in regions where the hydrostatic stress component is higher. The toughened PR-520 resin has a higher failure stress in tension and a higher maximum stress in shear compared to the untoughened E-862 resin.

The effect of strain rate on the shape of the stress-strain curves can be understood in terms of molecular structure and molecular motions by comparing the time scale (or rate) of molecular motions to the time scale (or rate) of deformation in a particular test. At high strain rates, deformation during the time scale of the test involve only short-range intermolecular interactions between polymer chains without any change in the intramolecular configuration (shape) of the large polymer chains. This type of deformation is not strongly rate dependent, as shown in figures 23 and 24 . At lower test rates molecular motions are fast enough (compared to the time scale of the test) to allow changes in the intramolecular configuration (i.e. shape of the extended polymer chain) during the test. Below the glass transition temperature, these changes in shape result from very short displacements of small segments of the polymer chains. These motions result in viscoelastic (or time-dependent) material behavior at all strain levels and contribute to plasticity at larger strains.

If the duration of a constant strain rate tensile test is sufficiently long, the shape of the stress-strain curve is affected by stress relaxation during the test, as shown in the stress relaxation curves at $30^{\circ} \mathrm{C}$ in figures 25 and 26. Since displacement is constant during the stress relaxation test, the data in figures 25 and 26 can be considered to be taken at zero strain rate. For the low strain rate tensile tests shown in figures 12 and 18, the duration of the tests is about 15 minutes or more. The data in figures 25 and 26 indicate that stress relaxation is significant during this time interval. The durations of the intermediate and high rate tests in figures 12 and 18 are too short for significant stress relaxation to occur. As a result, stress-strain curves at the intermediate and high strain rates are nearly linear until brittle failure occurs. In contrast, stress relaxation during the low rate tests prevents the stress from reaching the level required for brittle failure, and the stress-strain curves show a non-linear ductile response. As mentioned above, the same molecular motions responsible for stress relaxation (i.e. viscoelasticity) can also allow damage and plastic deformation to occur at higher strains. It is therefore not possible to separate the effects of viscoelasticity, plasticity, and damage on the shape of the stress-strain curves without further investigations such as determining the reversibility of the deformation and examining test specimens for indications of flow or damage.

The effect of strain rate on the shapes of the shear stress-strain curves in figures 11 and 17 can also be understood in terms of molecular motions. Similar to the tensile tests, the stress-strain curves are linear and nearly independent of rate in the low strain region where stiffness is determined primarily by intermolecular interactions and the time is too short for stress relaxation to be significant. However, in contrast to the tensile tests, the torsion specimens never fail in a brittle manner. Instead, yielding occurs at a low strain followed by a long plateau region of nearly constant stress. The strain at which the curves become nonlinear and the magnitude of the stress in the plateau region both increase with increasing 
strain rate. Both of these effects are consistent with a decrease in the amount of stress relaxation and plastic deformation as the time scale becomes shorter in the higher rate tests.

\section{Constitutive Modeling}

The constitutive model presented is based on Bodner's internal state variable constitutive equations (Bodner 2002), which were originally developed to analyze the viscoplastic deformation of metals above one-half of the melting temperature. The model is modified to analyze the strain rate and hydrostatic stress dependent, nonlinear deformation of polymeric materials. In the present model, a single unified strain variable is defined to represent all inelastic strains (Stouffer and Dame 1996). Inelastic strains are assumed to be present at all values of stress (there is no yield condition), and state variables, which evolve with stress and inelastic strain, are defined to represent the average effects of the deformation mechanisms. All of the nonlinearity and strain rate dependence is assumed to be due to inelastic deformation, where in reality the nonlinearity could be due to mixture of deformation and damage.

Flow and Evolution Equations. - Small strain theory is assumed to apply where the total strain rate, $\dot{\varepsilon}_{i j}$, is composed from elastic, $\dot{\varepsilon}_{i j}^{e}$, and inelastic, $\dot{\varepsilon}_{i j}^{I}$, parts:

$$
\dot{\varepsilon}_{i j}=\dot{\varepsilon}_{i j}^{e}+\dot{\varepsilon}_{i j}^{I}
$$

The elastic strain rate is given by Hooke's law, and the inelastic strain rate is written in the form:

$$
\dot{\varepsilon}_{i j}^{I}=\lambda \frac{\partial f}{\partial \sigma_{i j}}
$$

where $\lambda$ is a scalar rate function of the internal state variables, and $f$ is a flow potential. The flow potential $f$ is taken in a form proposed for polymers by Li and Pan (1990), Chang and Pan (1997), and Hsu, et al. (1999), which is based on the Drucker-Prager hydrostatic stress dependent yield criterion (see Khan and Huang (1995)):

$$
f=\sqrt{J_{2}}+\alpha \sigma_{k k}
$$

where $J_{2}=(1 / 2) S_{i j}$ is the second invariant of the deviatoric stress tensor, and $\alpha$ is an internal hydrostatic stress state variable. The scalar rate function $\lambda$ is taken in a form used by Bodner (2002):

$$
\lambda=2 D_{0} \exp \left[-\frac{1}{2}\left(\frac{Z}{\sigma_{e f}}\right)^{2 n}\right]
$$

where $D_{0}$ and $n$ are material constants, and $Z$ is an internal stress state variable. The effective stress $\sigma_{e f}$ is given by $\sigma_{e f}=\sqrt{3 J_{2}}+\sqrt{3} \alpha \sigma_{k k}$. With these definitions the inelastic work rate $\dot{W}^{I}$ is:

$$
\dot{W}^{I}=\sigma_{i j} \dot{\varepsilon}_{i j}^{I}=\sigma_{e f} \dot{\varepsilon}_{e q}^{I}
$$

where the equivalent inelastic strain rate, $\dot{\varepsilon}_{e q}^{I}$, can be determined to be $\dot{\varepsilon}_{e q}^{I}=\sqrt{(2 / 3) \dot{\varepsilon}_{i j}^{I} \dot{\varepsilon}_{i j}^{I}} / \sqrt{1+6 \alpha^{2}}$. The value of the scalar rate function $\lambda$ is related to the equivalent inelastic strain rate $\dot{\varepsilon}_{e q}^{I}$ by $\lambda=\sqrt{3} \dot{\varepsilon}_{e q}^{I}$. An explicit equation for the components of the inelastic strain rate is obtained by substituting the derivative of the flow potential (eq. (5)), and the scalar rate function $\lambda$ from equation (6) in equation (4): 


$$
\dot{\varepsilon}_{i j}^{I}=2 D_{0} \exp \left[-\frac{1}{2}\left(\frac{Z}{\sigma_{e f}}\right)^{2 n}\right]\left(\frac{S_{i j}}{2 \sqrt{J_{2}}}+\alpha \delta_{i j}\right)
$$

The evolution equations of the internal state variables $Z$ and $\alpha$ are:

$$
\begin{aligned}
& \dot{Z}=q\left(Z_{1}-Z\right) \dot{\varepsilon}_{e q}^{I} \\
& \dot{\alpha}=q\left(\alpha_{1}-\alpha\right) \dot{\varepsilon}_{e q}^{I}
\end{aligned}
$$

where $q$ is a material constant representing the "hardening" rate, and $Z_{1}$ and $\alpha_{1}$ are material constants representing the maximum value of $Z$ and $\alpha$, respectively. The initial values of $Z$ and $\alpha$ are defined by the material constants $Z_{0}$ and $\alpha_{0}$. The evolution equation for the state variable $\alpha$ is new to this work, and is assumed to take the same form as the evolution equation for the state variable $Z$. Equation (8) gives nonzero inelastic strains, and equations (9) and (10) give positive values for $\dot{Z}$ and $\dot{\alpha}$, when the material is loaded with hydrostatic stresses. In the original Bodner model, Bodner (2002), the inelastic work rate is used in the evolution equation for $Z$. In the present modeling it is found that using the equivalent inelastic strain rate gives better correlation with the data, and is also easier to use.

Determination of Material Constants. - The material constants that need to be determined include $D_{0}, n, Z_{0}, Z_{1}, \alpha_{0}, \alpha_{1}$, and $q$. The procedure to be used is summarized here, and more details can be found in Goldberg, et al. (2003). The constants are determined using shear and tensile stress-strain curves from constant strain rate tests. The value of $D_{0}$ is assumed to be equal to $10^{6} \mathrm{~s}^{-1}$, which is $10^{4}$ times the maximum applied strain rate. The value of $n$ and $Z_{1}$ is determined from shear tests. For pure shear equation $(8)$ reduces to:

$$
\dot{\gamma}^{I}=2 D_{0} \exp \left[-\frac{1}{2}\left(\frac{Z}{\sqrt{3} \tau}\right)^{2 n}\right]
$$

The hydrostatic stress component is not present which means that the variable $\alpha$, and consequently the constants $\alpha_{1}$ and $\alpha_{0}$, that are associated with the effects of the hydrostatic stress, are absent from the equation. In shear, the experimental results show a ductile material response with stress-strain curves that display a defined "saturation" stress where the inelastic strain rate equals the total applied strain rate. This occurs at the point where the stress-strain curve flattens out and becomes horizontal. In equation (11), when the shear stress reaches "saturation", the value of $Z$ is $Z_{1}$. The value of $n$ and $Z_{1}$ is determined from equation (11) by using the experimental values of the "saturation" shear stress at different strain rates (least squares regression of a linearized form of eq. (11)).

To determine the value of $Z_{0}$, equation (11) is rearranged into the form:

$$
Z=\left[-2 \ln \left(\frac{\dot{\gamma}^{I}}{2 D_{0}}\right)\right]^{\frac{1}{2 n}} \sqrt{3} \tau
$$

The value of $Z_{0}$ is calculated by identifying the value of the shear stress where the stress-strain curve becomes nonlinear for a particular constant strain rate shear test. The point where the stress-strain curve becomes nonlinear is defined as the approximate point where the curve appreciably deviates from a linear extrapolation of the initial stress-strain curve. The total shear strain rate divided by 100 was found by trial 
and error to approximate the inelastic strain rate at this point reasonably well. Using the data from the lowest strain rate test available has been found to give adequate values of $Z_{0}$. However, the determination can also be made by averaging the values of $Z_{0}$ that are obtained from data from all the available strain rates.

To determine the value of the constant $q$ in equations (9) and (10), first equation (9) is integrated for the case of pure shear loading:

$$
Z=Z-\left(Z_{1}-Z_{0}\right) \exp \left(\frac{-q}{\sqrt{3}} \gamma^{I}\right)
$$

where $\gamma^{I}$ is the inelastic shear strain. At saturation, the value of $Z$ approaches $Z_{1}$, resulting in the exponential term approaching zero. Assuming that saturation occurs when the exponential term is equal to 0.01gives:

$$
\exp \left(\frac{-q}{\sqrt{3}} \gamma_{s}^{I}\right)=0.01
$$

For a given value of $\gamma_{s}^{I}$, the inelastic shear strain at saturation, equation (14) can be solved for $q$. If $\gamma_{s}^{I}$ is found to vary with strain rate, the parameter $q$ is computed at each strain rate and regression techniques are utilized to determine an expression for the variation of $q$. Since Equations (9) and (10) have the same formats, integrating and using equation (10) would lead to an identical value for $q$.

The value of the constants $\alpha_{0}$ and $\alpha_{1}$ are obtained from the requirement that the effective stress $\sigma_{e f}$ $\left(\sigma_{e f}=\sqrt{3 J_{2}}+\sqrt{3} \alpha \sigma_{k k}\right)$, has the same value in uniaxial tension and in shear tests at the point where the stress-strain curve becomes nonlinear and at the point where the curves become saturated. At these two points the effective stress is given by:

$$
\begin{gathered}
\sigma_{n l}\left(1+\sqrt{3} \alpha_{0}\right)=\sqrt{3} \tau_{n l} \\
\sigma_{s}\left(1+\sqrt{3} \alpha_{1}\right)=\sqrt{3} \tau_{s}
\end{gathered}
$$

where $\sigma_{n l}$ and $\tau_{n l}$ are the tensile and shear stresses at the point where the respective stress-strain curves become nonlinear, and $\sigma_{s}$ and $\tau_{s}$ are the tensile and shear stresses at saturation, respectively. It is assumed that the constants $\alpha_{0}$ and $\alpha_{1}$ are strain rate independent.

Modeling the PR-520 resin.-The ability of the developed constitutive equations to correctly simulate the strain rate and hydrostatic stress dependent response of polymers is demonstrated for the PR-520 resin. The material constants $D_{0}, n, Z_{0}, Z_{1}, \alpha_{0}, \alpha_{1}$, and $q$. were determined according to the procedures outlined in the previous subsection. The elastic modulus for the low and medium strain rate is estimated from the corresponding stress-strain curves. For the high strain rate, since the initial part of the stress-strain curve is not accurate, the elastic modulus is estimated by extrapolating the stress strain curves to zero. The values of all the constants are listed in table 1 .

The computed shear stress-shear strain curves for the low, medium, and high strain rates, are shown together with the experimental results in figure 17 (in the low strain rate it is difficult to see the calculated curve since it coincides with several of the overlapping experimental curves). Overall, the computed results correlate well with the experimental values for all strain rates. Specifically, the nonlinearity and rate dependence of the experimental results are captured qualitatively, and the quantitative match between the experimental and computed results is reasonably good. 
The computed tensile stress-strain curves for all three strain rates, along with the experimental results for comparison, are shown in figure 18. Once again, qualitatively the nonlinearity and rate dependence of the experimental results is captured. Quantitatively, at the medium strain rate the stresses in the nonlinear range are somewhat under predicted, but otherwise there is a good correlation between the experimental and computed curves. The high strain rate tensile stresses are somewhat under predicted at lower strains, but this is most likely due to the inaccuracy of the SHB technique at small strains. For the tensile results, the important point to note is that the material constants were primarily computed using the shear data, and the comparison of the computed results to the experimental tensile data is overall reasonably good.

To further explore the significance of properly accounting for the hydrostatic stress effects in the analysis, the tensile stress-strain curve at the low strain rate is once again considered. Figure 27 shows the experimental stress-strain curves and the computed curve when the hydrostatic stress effects are included in the model ( $\alpha$ included in the constitutive model), and the computed curve when the hydrostatic stress effects are not included in the model ( $\alpha$ not included in the constitutive model). In the latter case the calculations were carried out with $\alpha_{0}=\alpha_{1}=0$. As can be seen in the figure, when the hydrostatic stress effects are neglected in computing the tensile response, the stresses are significantly over predicted as compared to the experimental results, which indicates that the hydrostatic stress effects are significant for polymers and must be accounted for within an analysis.

\section{Conclusions}

The tensile and shear properties of E-862 and PR-520 epoxy resins were measured over a range of strain rates from approximately $5 \times 10^{-5} \mathrm{~s}^{-1}$ to $700 \mathrm{~s}^{-1}$. The toughened PR-520 resin has a higher failure stress in tension and a higher maximum stress in shear compared to the untoughened E-862 resin. In tension, the hydrostatic component of the stress causes failure of the untoughened resin outside of the gage section. Premature failure occurs near the location of adhesively bonded stain gages. The toughened resin is not sensitive to these premature failure effects in tension. Localized deformation in the PR-520 resin causes a decreasing slope in the stress-strain curves at high strains in the shear tests. In the tensile tests, the transition from ductile to brittle response occurs between the low and intermediate test rates examined. The ductile response is attributed to the effects of stress relaxation and plastic deformation that can occur during the relatively long time scale of the lowest rate tests. In the shear tests the material response is ductile at all strain rates. The onset of nonlinearity and the magnitude of the maximum stress decrease with decreasing strain rate. These effects are also attributed to the relatively larger effect of stress relaxation and plastic deformation during the longer time scale of the low rate tests. The hydrostatic stress has a significant effect on the response (load-deformation relationship) of the resins. These effects must be accounted for in any constitutive formulation that is used to model the material response. A unified internal state variable formulation that is based on Bodner's formulation, and is modified to include the effects of the hydrostatic stress, is introduced. Good correlation with the experimental results on the PR-520 epoxy is obtained.

\section{References}

Bodner, S.R., (2002) Unified Plasticity for Engineering Applications, Kluwer Academic/Plenum Publishers, New York.

Bordonaro, C.M., (1995) Rate Dependent Mechanical Behavior of High Strength Plastics: Experiment and Modeling, PhD thesis, Rensselaer Polytechnic Institute, Troy, New York.

Buckley, C.P., Harding, J., Hou, C., Ruiz, and C., Trojanowski, A. (2001) Deformation of Thermosetting

Resins at Impact Rates of Strain. Part I: Experimental Study, Journal of the Mechanics and Physics of Solids, vol. 49, pp. 1517-1538. 
Chang, W.J., and Pan, J., (1997) Effects of Yield Surface Shape and Round-Off Vertex on Crack-Tip Fields for Pressure-Sensitive Materials, International Journal of Solids and Structures, vol. 34, pp. 3291-3320.

Chen, W., and Zhou, B. (1998) Constitutive Behavior of Epon 828/T-403 at Various Strain Rates, Mechanics of Time-Dependent Materials, vol. 2, pp. 103-111.

Gilat, A. (2000) Torsional Kolsky Bar Testing, ASM Metals Handbook, Volume 8, Mechanical Testing and Evaluation, American Society of Metals, Materials Park, OH, pp 505-515.

Gilat, A., Krishna, K. (1977) The Effects of Strain Rate and Thickness on the Response of Thin Layers of Solder Loaded in Pure Shear, ASME Journal of Electronic Packaging, vol. 19, 1977, pp. 81-84.

Goldberg, R.K., Roberts, G.D., and Gilat, A., (2003) Implementation of an Associative Flow Rule Including Hydrostatic Stress Effects Into the High Strain Rate Deformation Analysis of Polymer Matrix Composites, NASA/TM-2003-212382, National Aeronautics and Space Administration, Washington, D.C.

Hou, J.P., Ruiz, C., and Trojanowski, A. (2000) Torsion Tests of Thermosetting Resins at Impact Strain Rate and Under Quasi-Static Loading, Materials Science \& Engineering A283, pp.181 and 188.

Hsu, S.-Y., Vogler, T.J., and Kyriakides, S., (1999) Inelastic behavior of an AS4/PEEK composite under combined transverse compression and shear, Part II: modeling, International Journal of Plasticity, vol. 15 , pp. 807-836.

Khan, A.S., and Huang, S., (1995) Continuum Theory of Plasticity, John Wiley and Sons, New York.

Kody, R.S., and Lesser, A.J. (1977) Deformation and Yield of Epoxy Networks in Constrained States of Stress, Journal of Materials Science, vol. 32, pp. 5637-5643.

Kolsky, H. (1949) An investigation of the Mechanical Properties of Materials at Very High Rates of Loading, Proc Phys Soc, London, vol. 62-B, pp. 676-700.

Krempl, E., and Ho, K., (2000) An Overstress Model for Solid Polymer Deformation Behavior Applied to Nylon 66, Time Dependent and Nonlinear Effects in Polymers and Composites, ASTM STP 1357, R.A. Schapery and C.T. Sun, eds., American Society for Testing and Materials, West Conshohocken, PA, pp. 118-137.

Li, F.Z., and Pan, J., (1990) Plane-Stress Crack-Tip Fields for Pressure-Sensitive Dilatant Materials, Journal of Applied Mechanics, vol. 57, pp. 40-49.

Shah Khan, M.Z., Simpson, G., and Townsend, C.R. (2002) A Comparison of the Mechanical Properties in Compression of Two Resin Systems, Materials Letters, vol. 52, pp. 173-179.

Staab, G.H., and Gilat, A. (1995) High strain Rate Response of Angle-Ply Glass/Epoxy Laminate, Journal of Composite Materials. vol. 29, 1995, pp. 1308-1320.

Stouffer, D.C., and Dame, L.T., (1996) Inelastic Deformation of Metals, Models, Mechanical Properties and Metallurgy, John Wiley and Sons, New York.

Walley, S.M., Field, J.E., Pope, P.H., and Safford, N.A. (1989) A Study of the Rapid Deformation Behaviour of a Range of Polymers, Phil. Trans. Royal Soc. London, Ser. A, vol. 328, no. 1597, pp. 1-33.

Ward, I.M., (1983) Mechanical Properties of Solid Polymers, John Wiley and Sons, New York.

Wineman, A.S., and Rajagopal, K.R. (2000) Mechanical Response of Polymers, Cambridge University Press, New York.

Zhang, C. and Moore, I.D. (1997) Nonlinear Mechanical Response of High Density Polyethylene, Part II: Uniaxial Constitutive Model, Polymer Engineering \& Science, vol. 37, pp. 414-420. 
TABLE 1.-MATERIAL CONSTANTS.

\begin{tabular}{|c|c|c|c|c|c|c|c|c|c|c|}
\hline Material & $\begin{array}{c}\text { Strain } \\
\text { Rate } \\
\mathrm{s}^{-1}\end{array}$ & $\begin{array}{c}\text { Modulus } \\
\mathrm{GPa}\end{array}$ & $\begin{array}{c}\text { Poisson's } \\
\text { Ratio }\end{array}$ & $\begin{array}{c}D_{0} \\
\mathrm{~s}^{-1}\end{array}$ & $n$ & $\begin{array}{c}Z_{0} \\
\mathrm{MPa}\end{array}$ & $\begin{array}{c}Z_{1} \\
\mathrm{MPa}\end{array}$ & $q$ & $\alpha_{0}$ & $\alpha_{1}$ \\
\hline \multirow{2}{*}{ PR-520 } & $7 \times 10^{-5}$ & 3.54 & 0.38 & $1 \times 10^{6}$ & 0.93 & 396.09 & 753.82 & 279.26 & 0.568 & 0.126 \\
\cline { 2 - 9 } & 1.76 & 3.54 & & & & & & & \\
\cline { 2 - 10 } & 420 & 7.18 & & & & & & & & \\
\hline
\end{tabular}

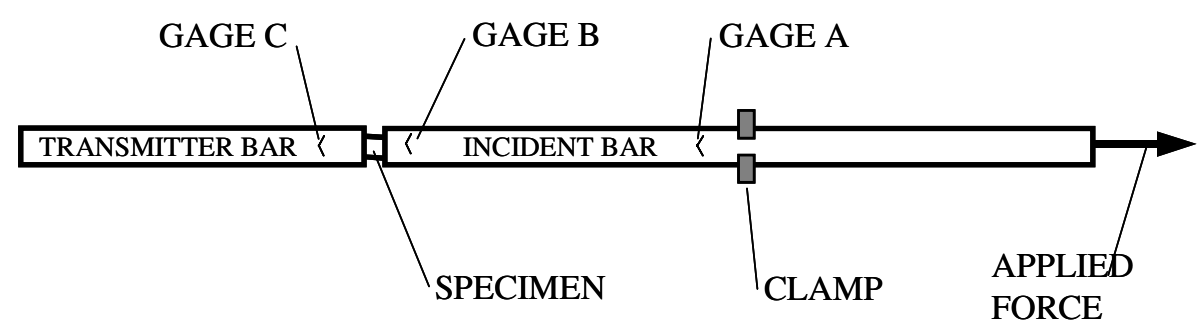

(a)

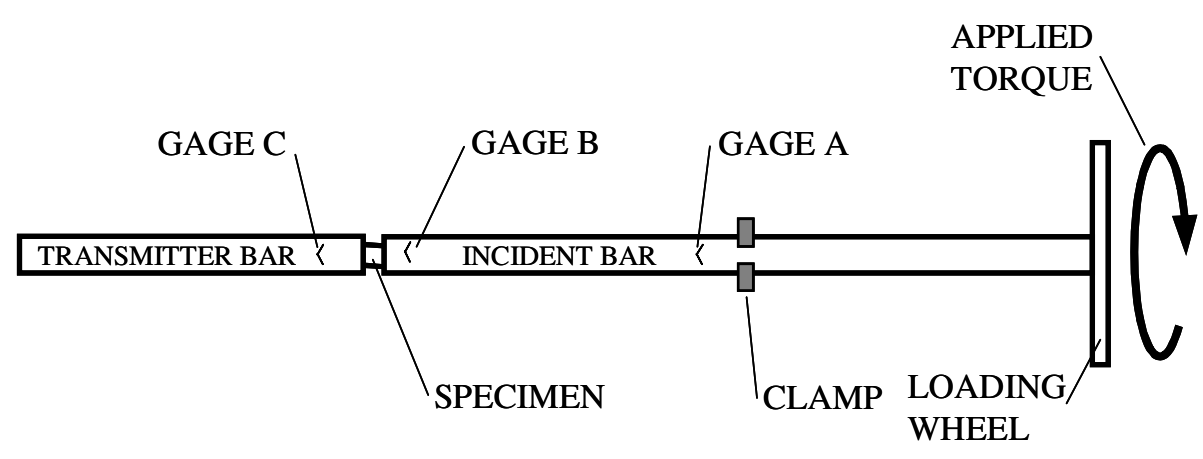

(b)

Figure 1.-Schematic of the tensile split Hopkinson bar apparatus, (a) tensile, (b) torsion. 

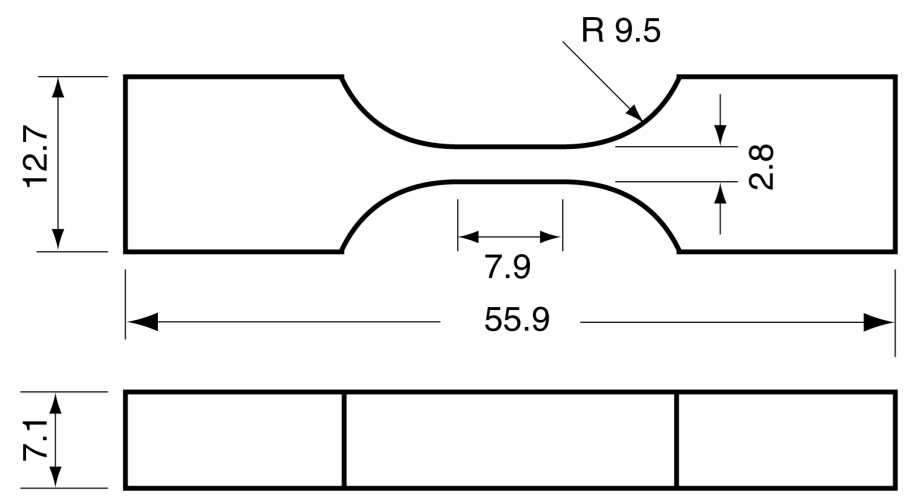

(a)

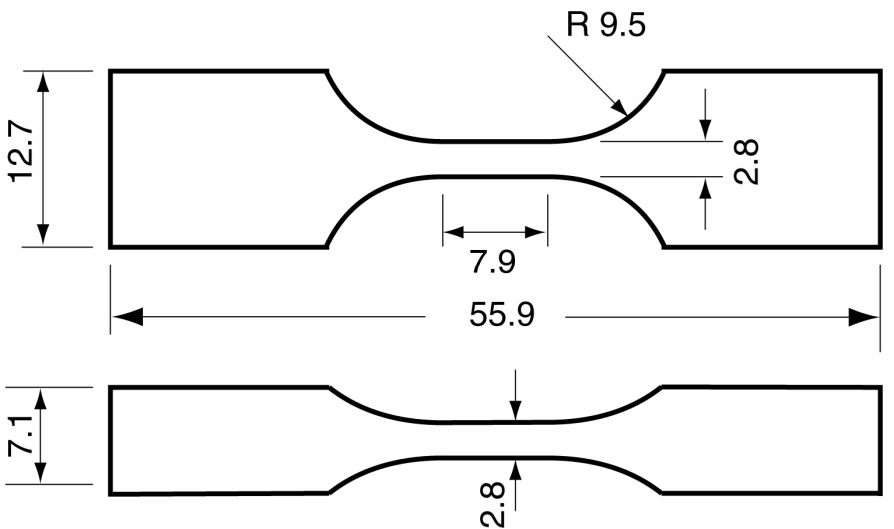

(b)

Figure 2.-Dog-bone shaped tensile test specimen (dimensions in $\mathrm{mm}$ ),

(a) specimen with uniform thickness, (b) specimen with reduced cross sectional area.

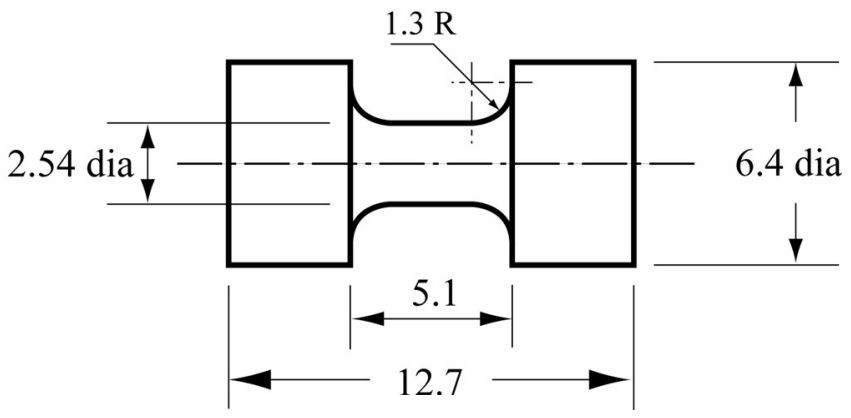

Figure 3.- Short cylindrical specimen and adapter for the tensile SHB tests (dimensions in $\mathrm{mm}$ ). 


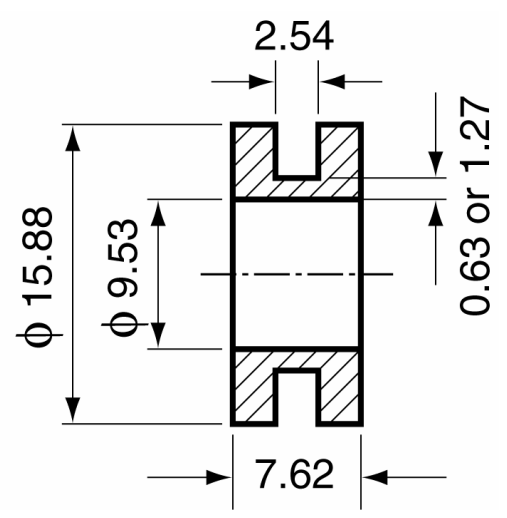

Figure 4.- Torsion test specimen (dimensions in $\mathrm{mm}$ ).

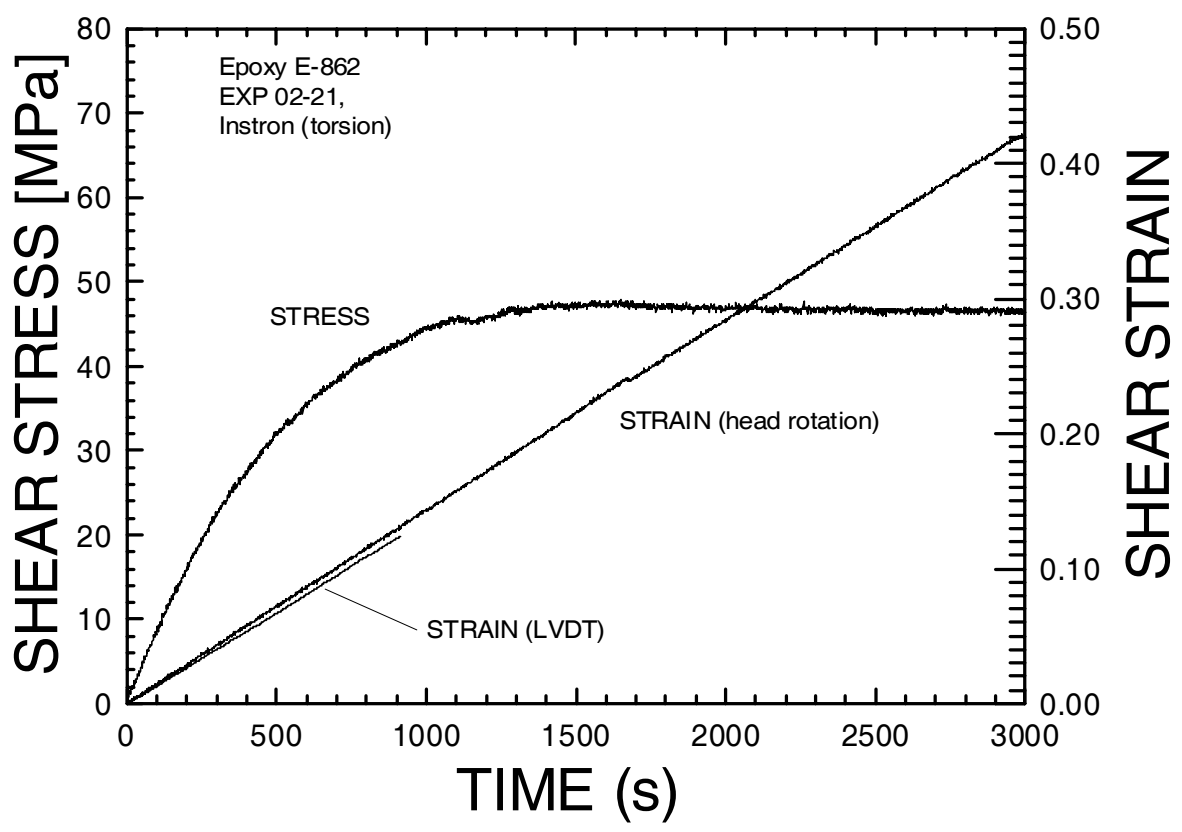

Figure 5.- Recorded data in a quasi-static torsion test. 

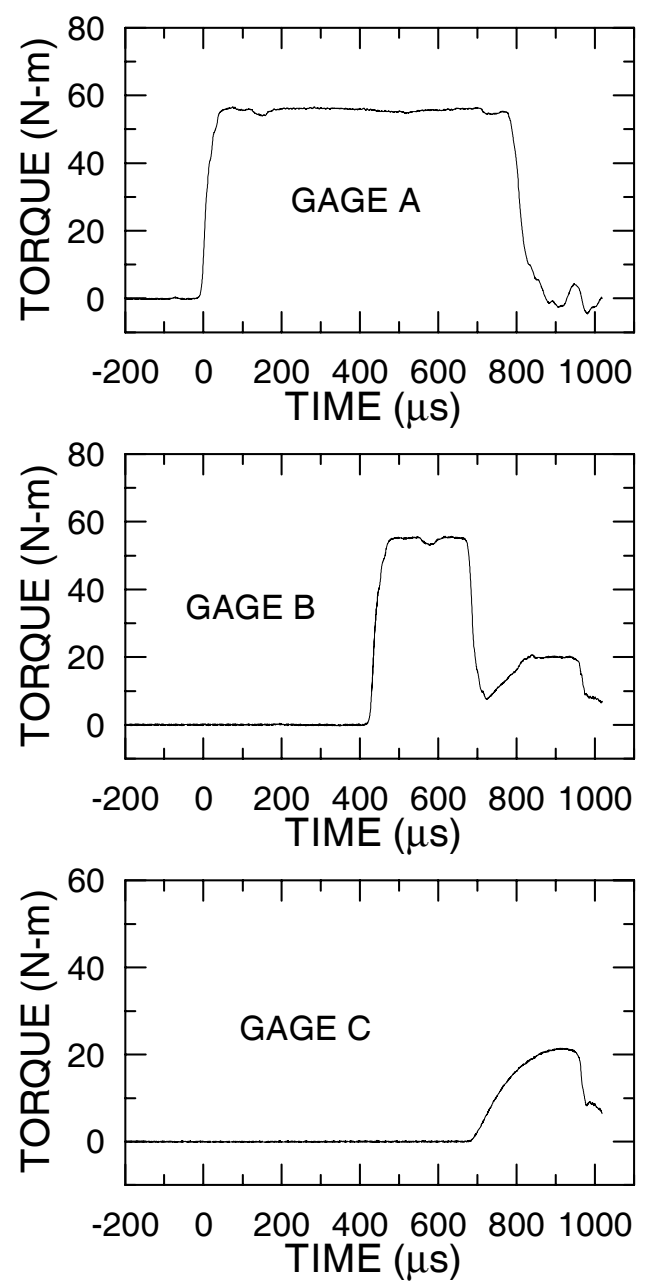

Figure 6.- Recorded data in a torsional SHB test. 


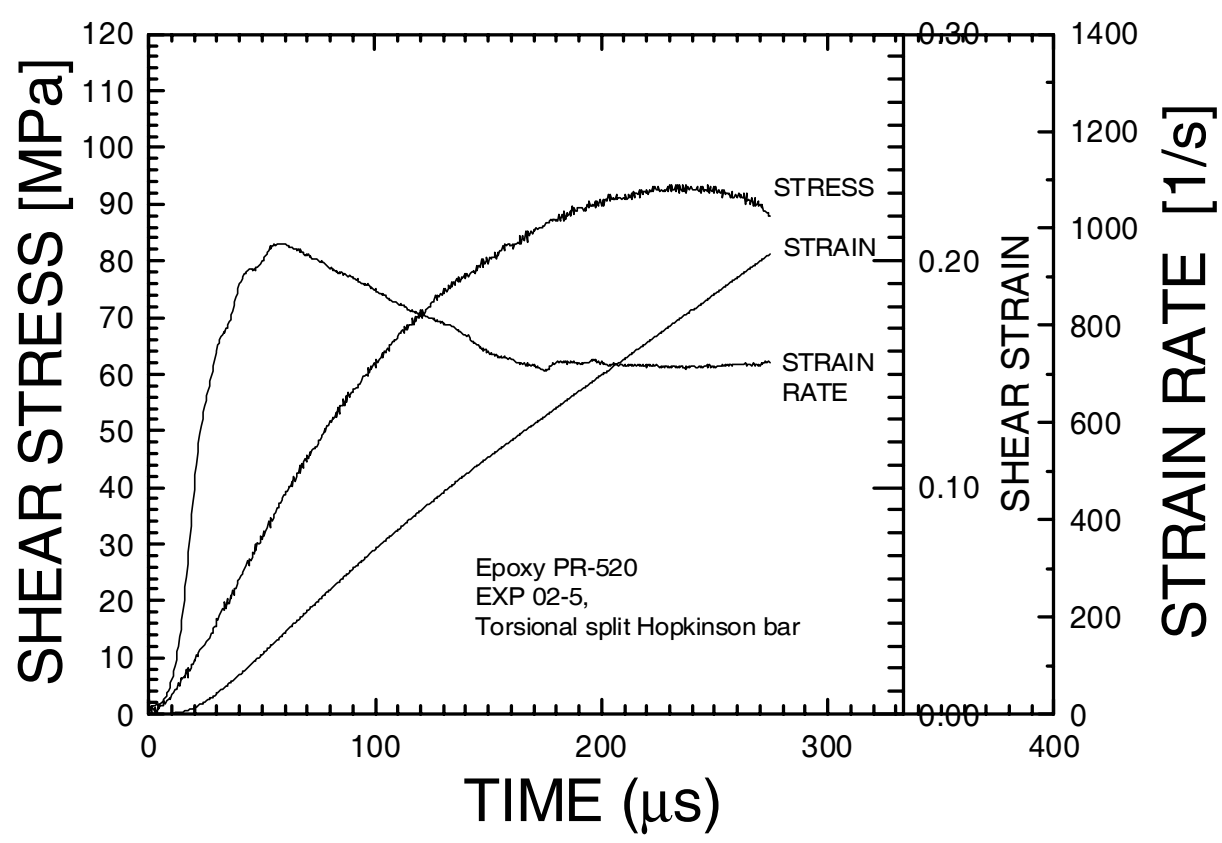

Figure 7.- - Stress, strain rate, and strain in a specimen during a torsional SHB test.

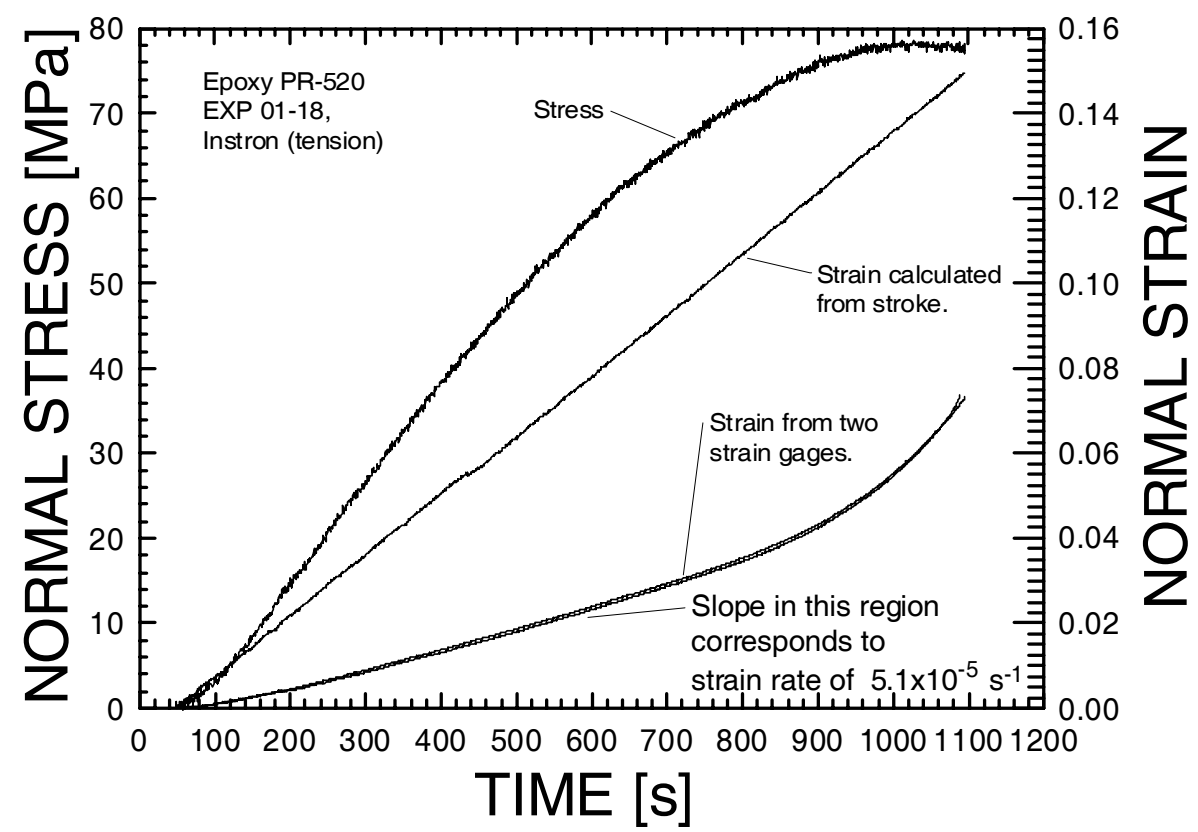

Figure 8.-Recorded data in a quasi-static tension test. 

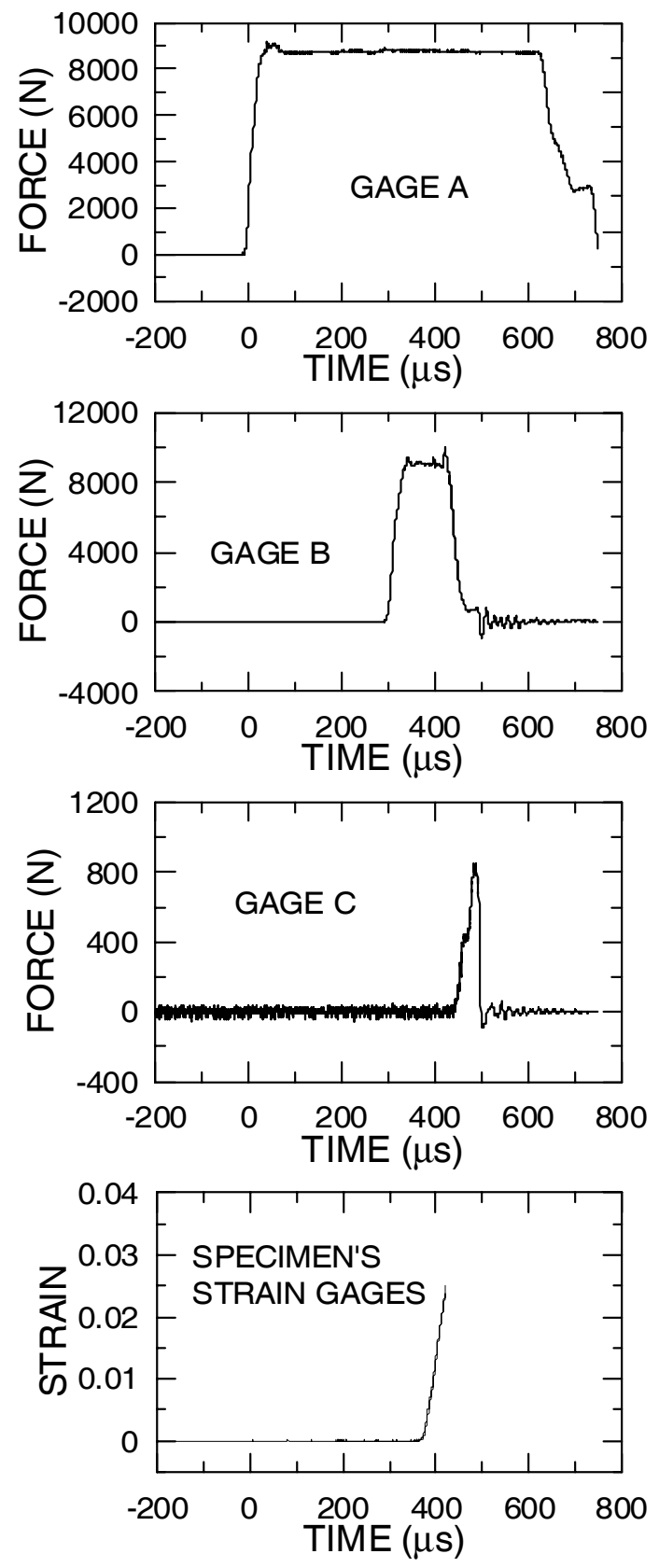

Figure 9.- Recorded data in a tensile SHB test. 


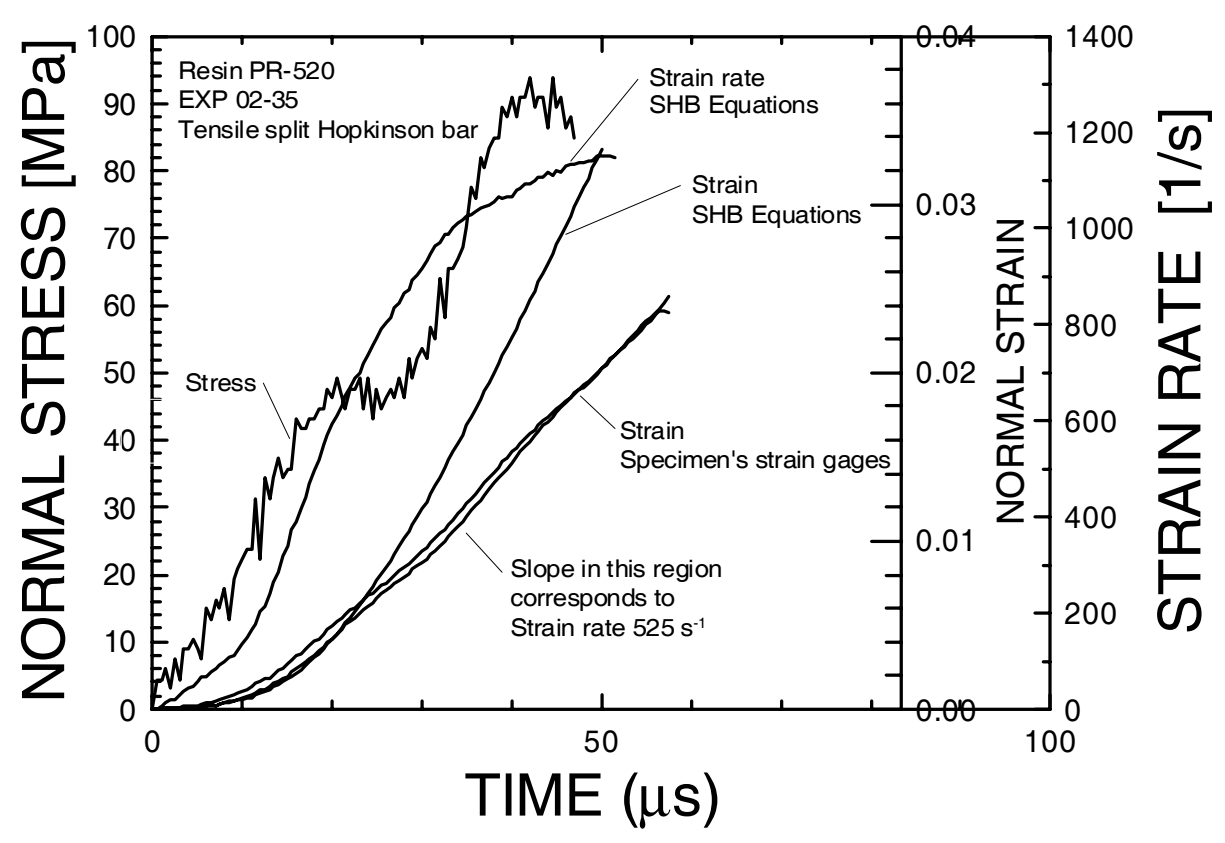

Figure 10.- Stress, strain rate, and strain in a specimen during a tensile SHB test.

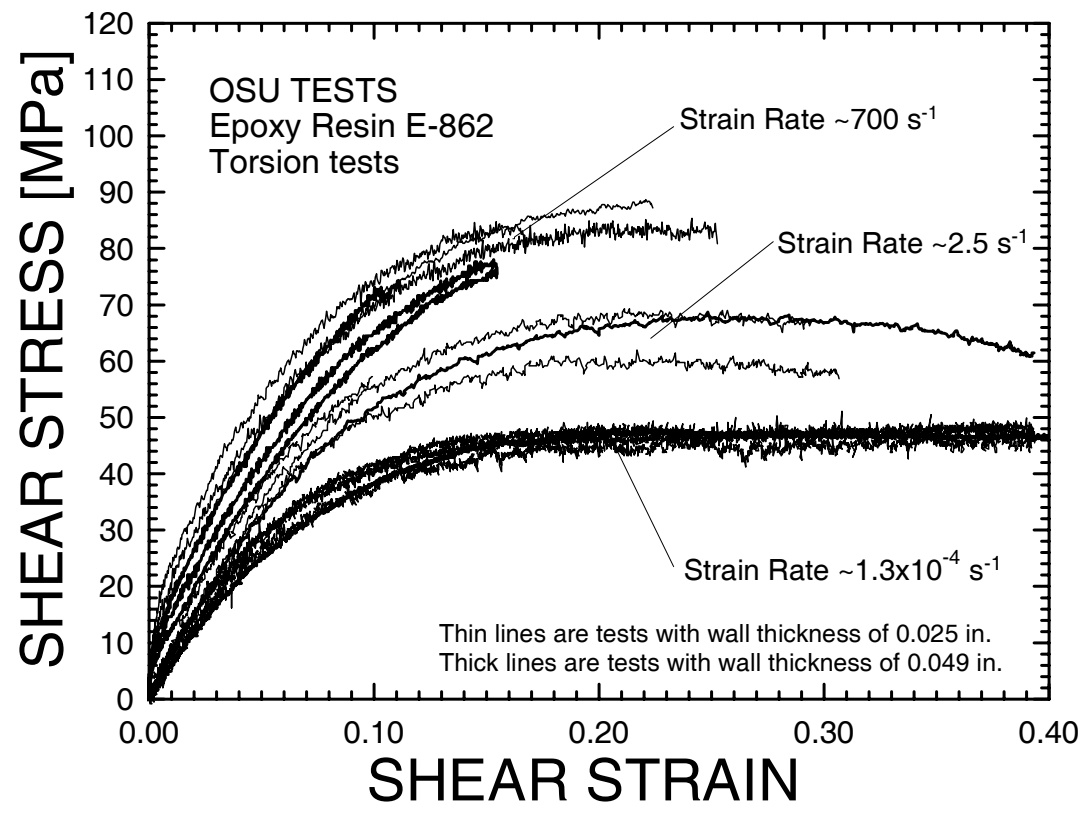

Figure 11.- Shear stress strain curves for E-862 resin at different strain rates. 


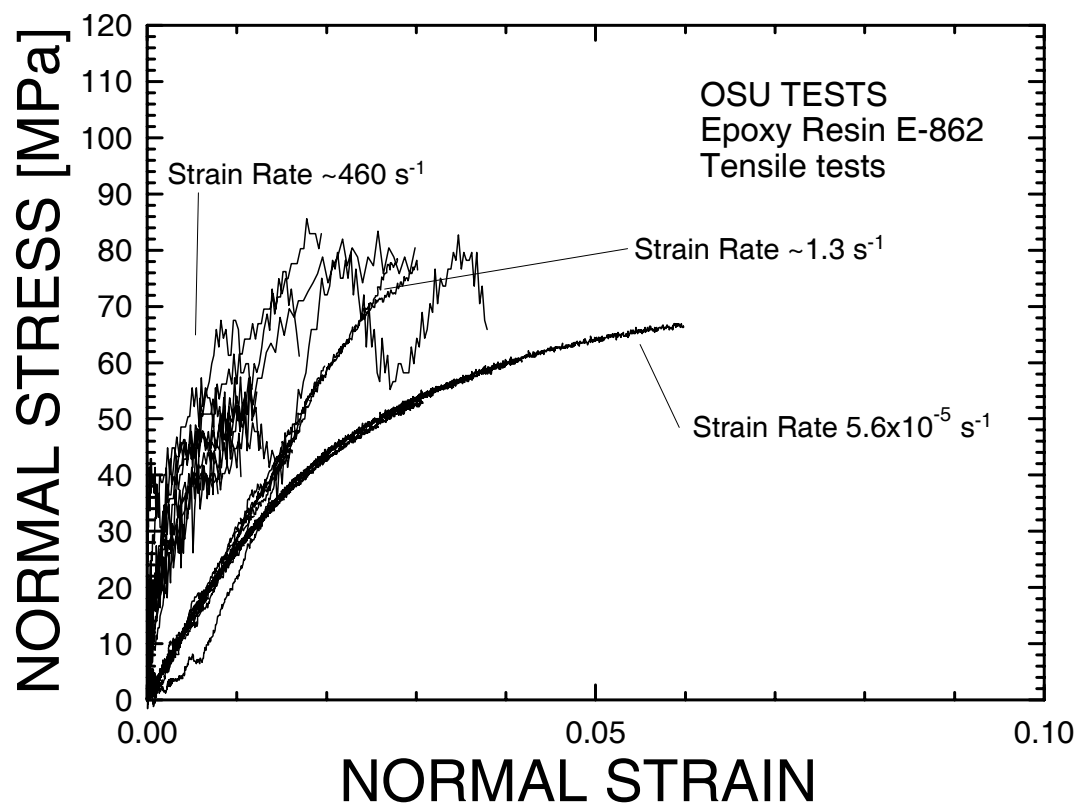

Figure 12.-Tensile stress strain curves for E-862 resin at different strain rates. 


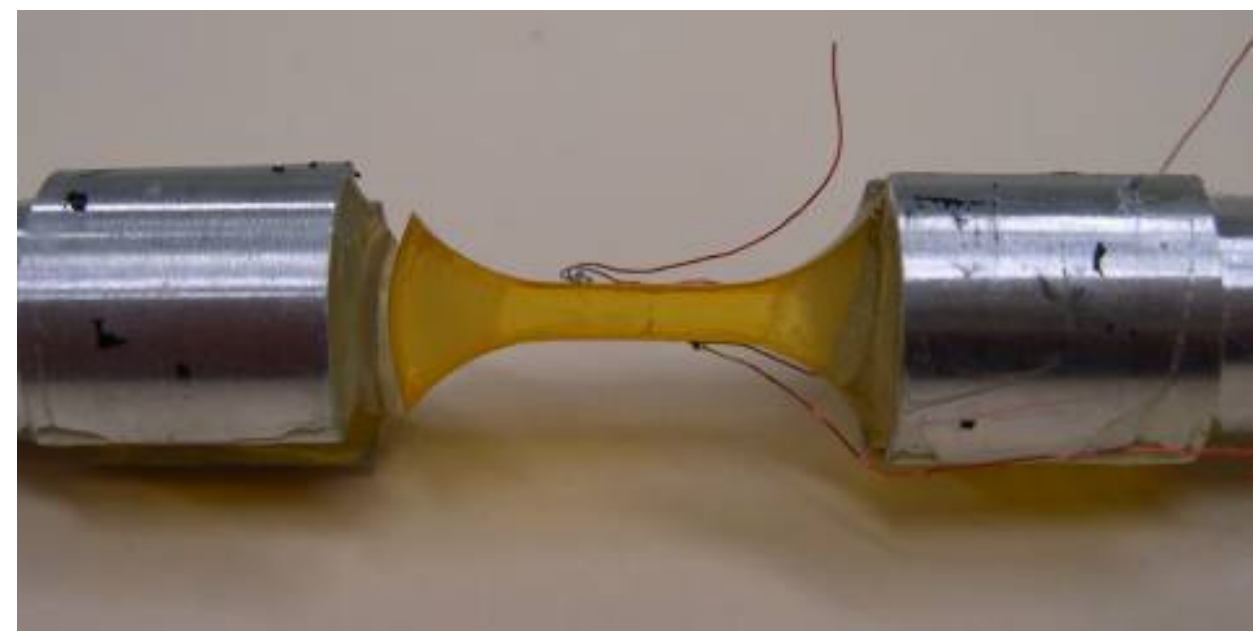

(a)

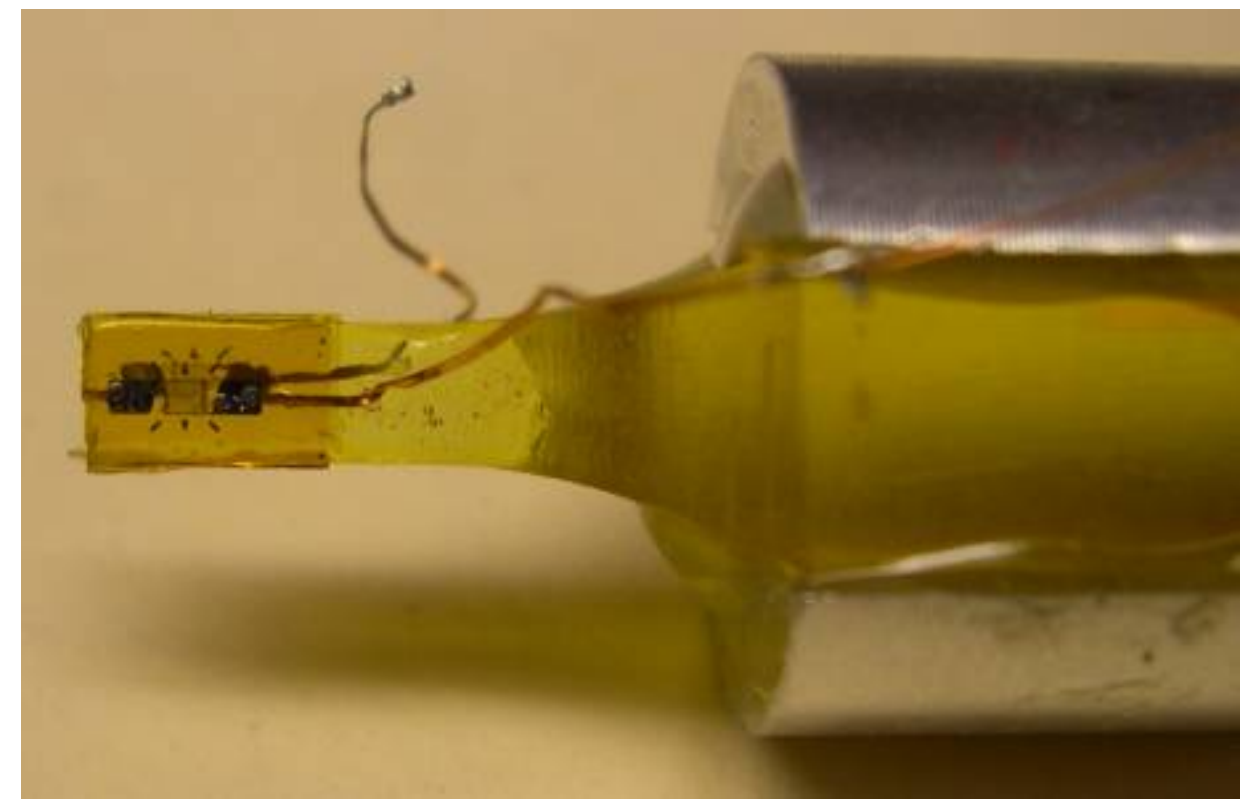

(b)

Figure 13.-Fractured E-862 resin specimens. 

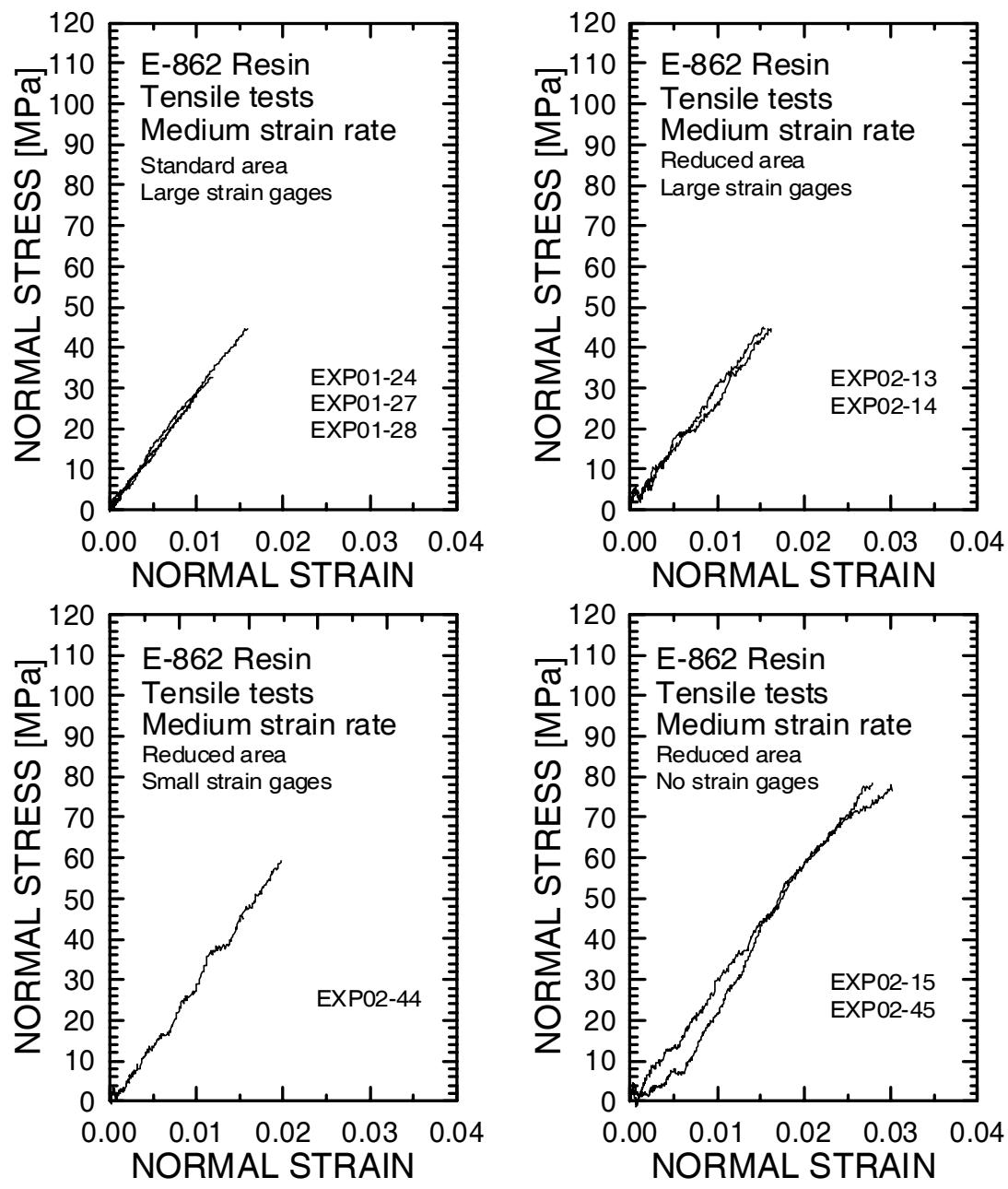

Figure 14.-Tensile stress strain curves for E-862 resin from medium strain rate experiments. 

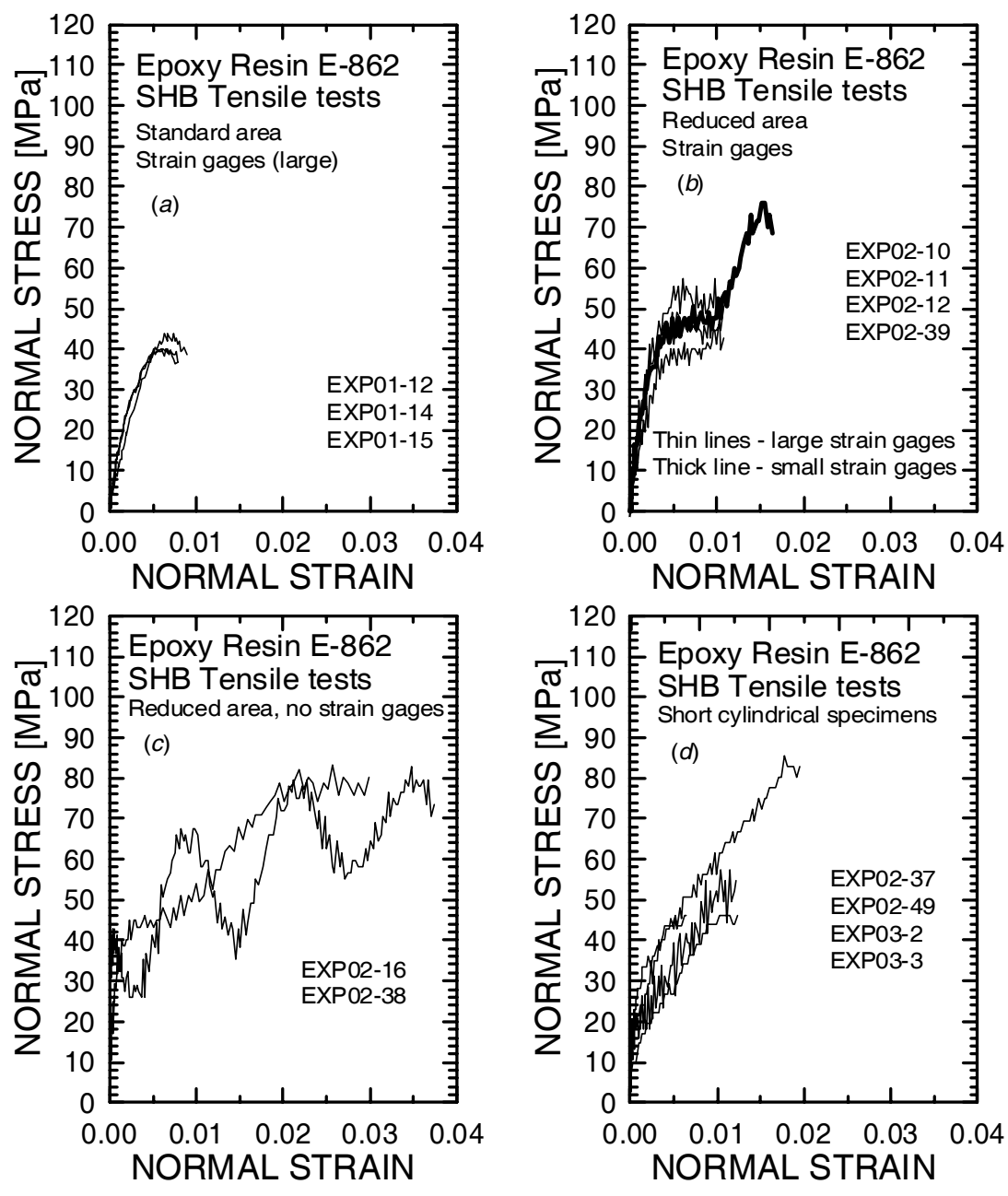

Figure 15.-Tensile stress strain curves for E-862 epoxy from split Hopkinson bar experiments. 


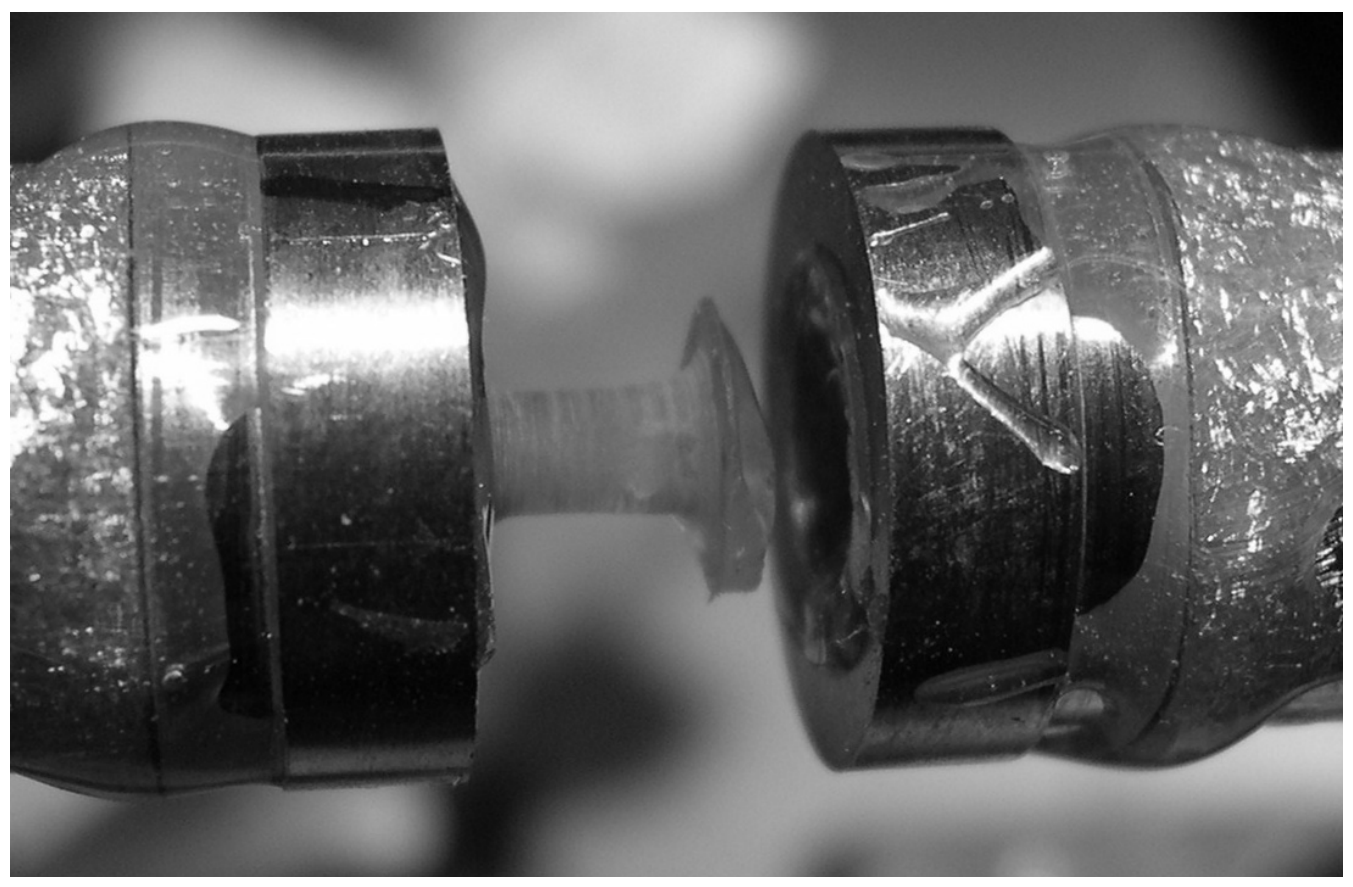

Figure 16.-Fractured E-862 resin specimens 


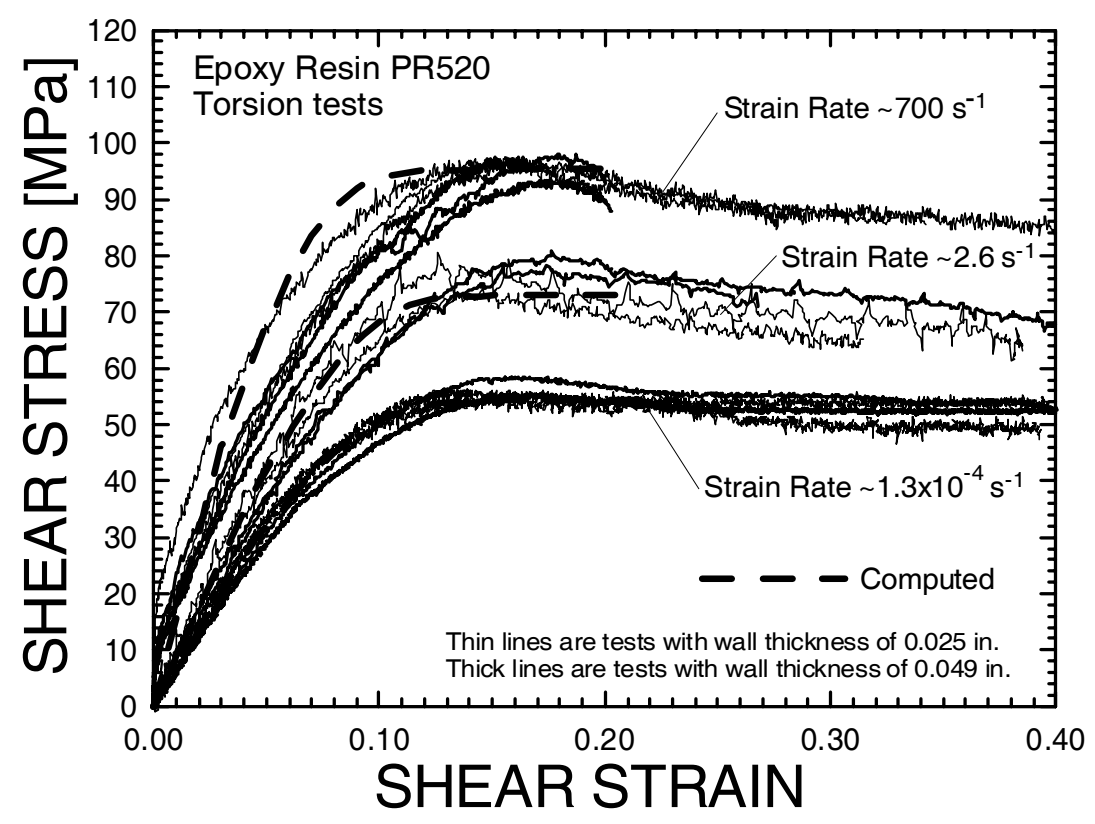

Figure 17.- Shear stress strain curves for PR-520 epoxy at different strain rates.

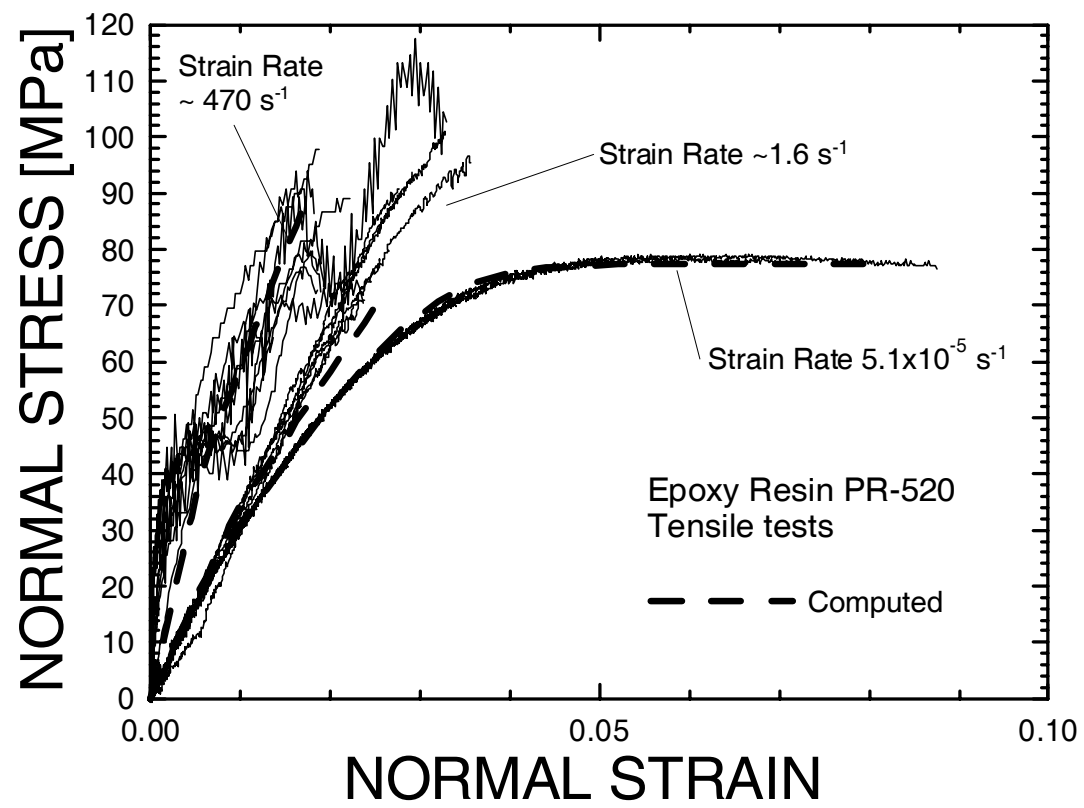

Figure 18.-Tensile stress strain curves for PR-520 epoxy at different strain rates. 

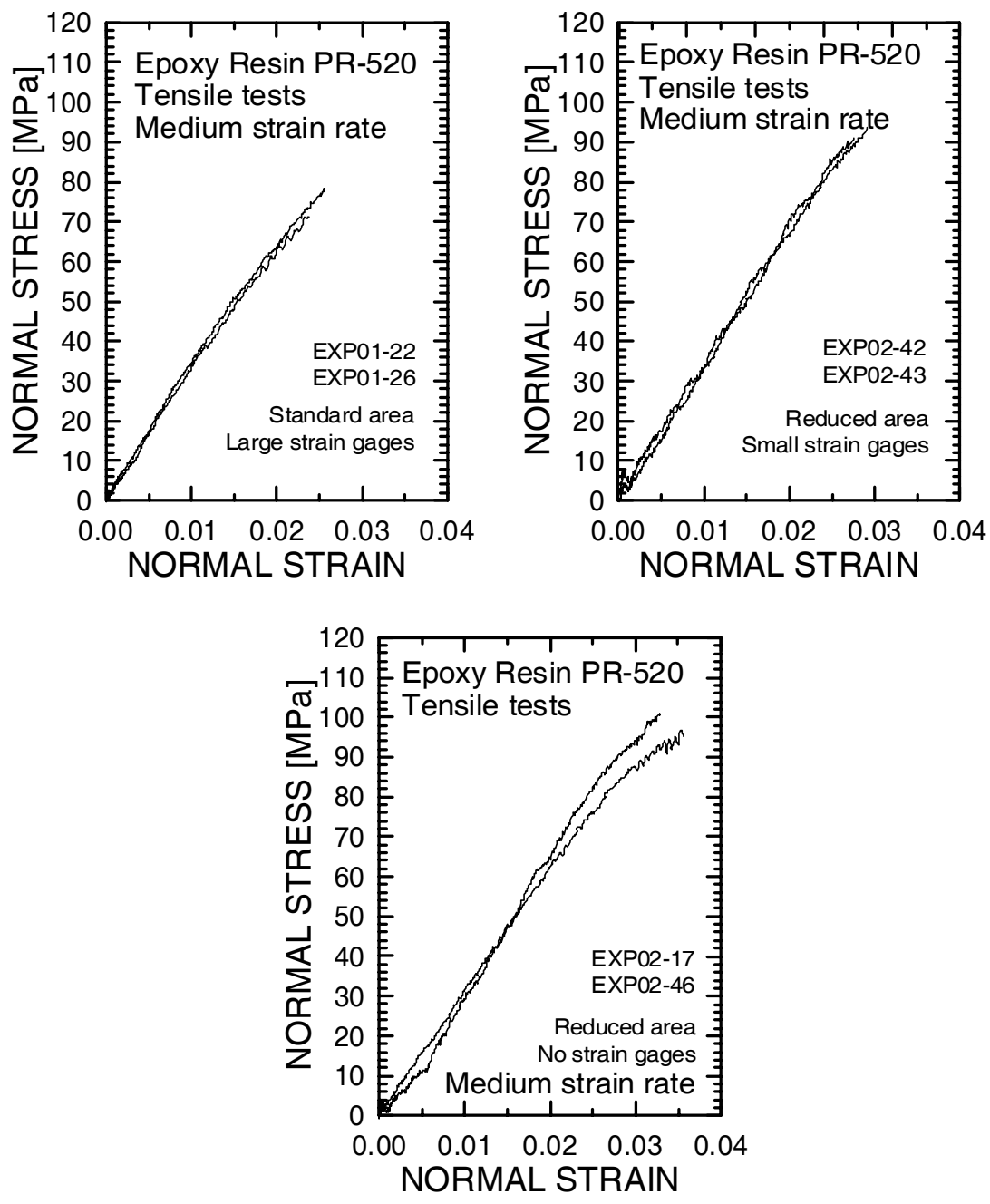

Figure 19.-Tensile stress strain curves for PR-520 epoxy from medium strain rate experiments. 

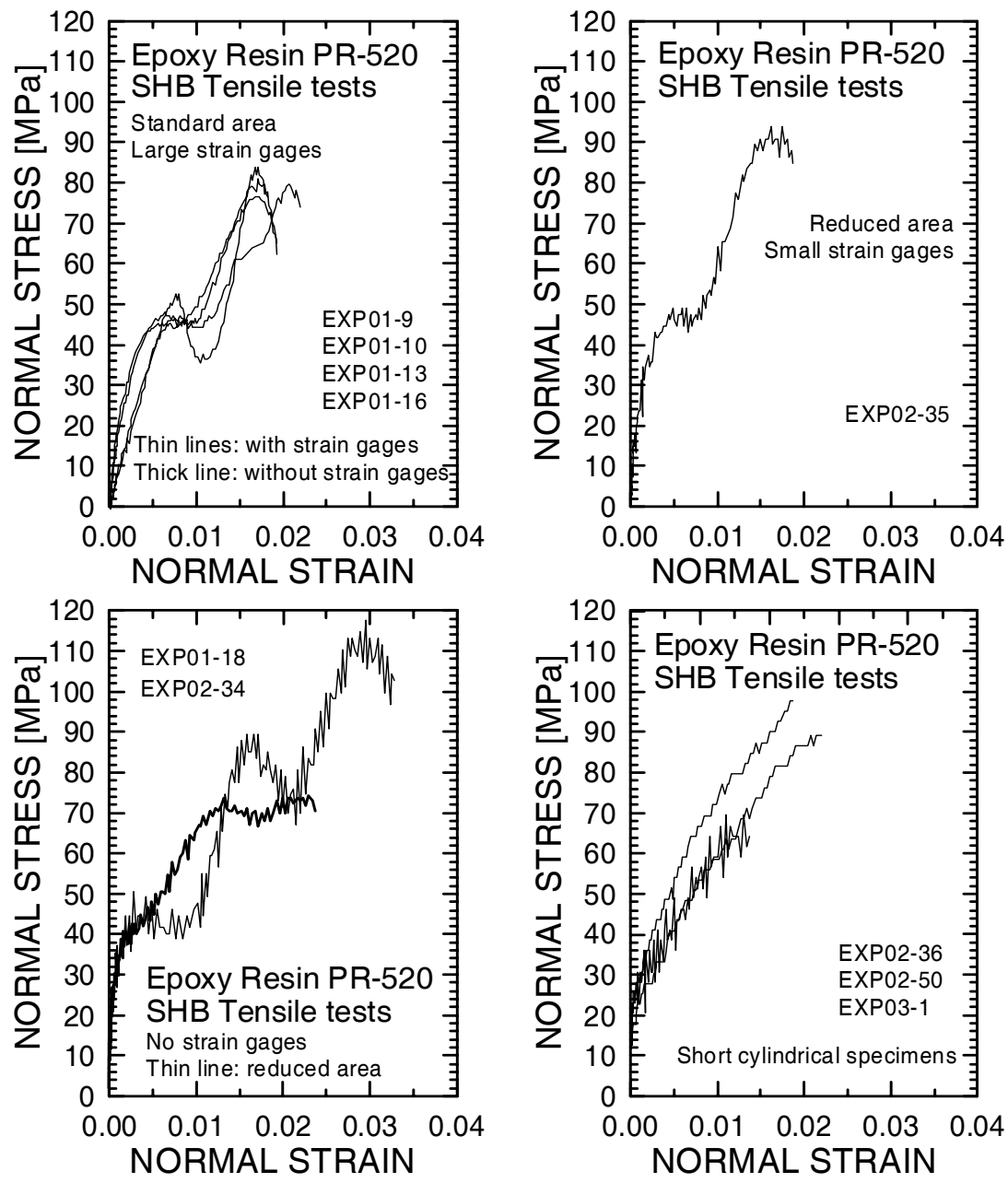

Figure 20.-Tensile stress strain curves for PR-520 epoxy from split Hopkinson bar experiments. 


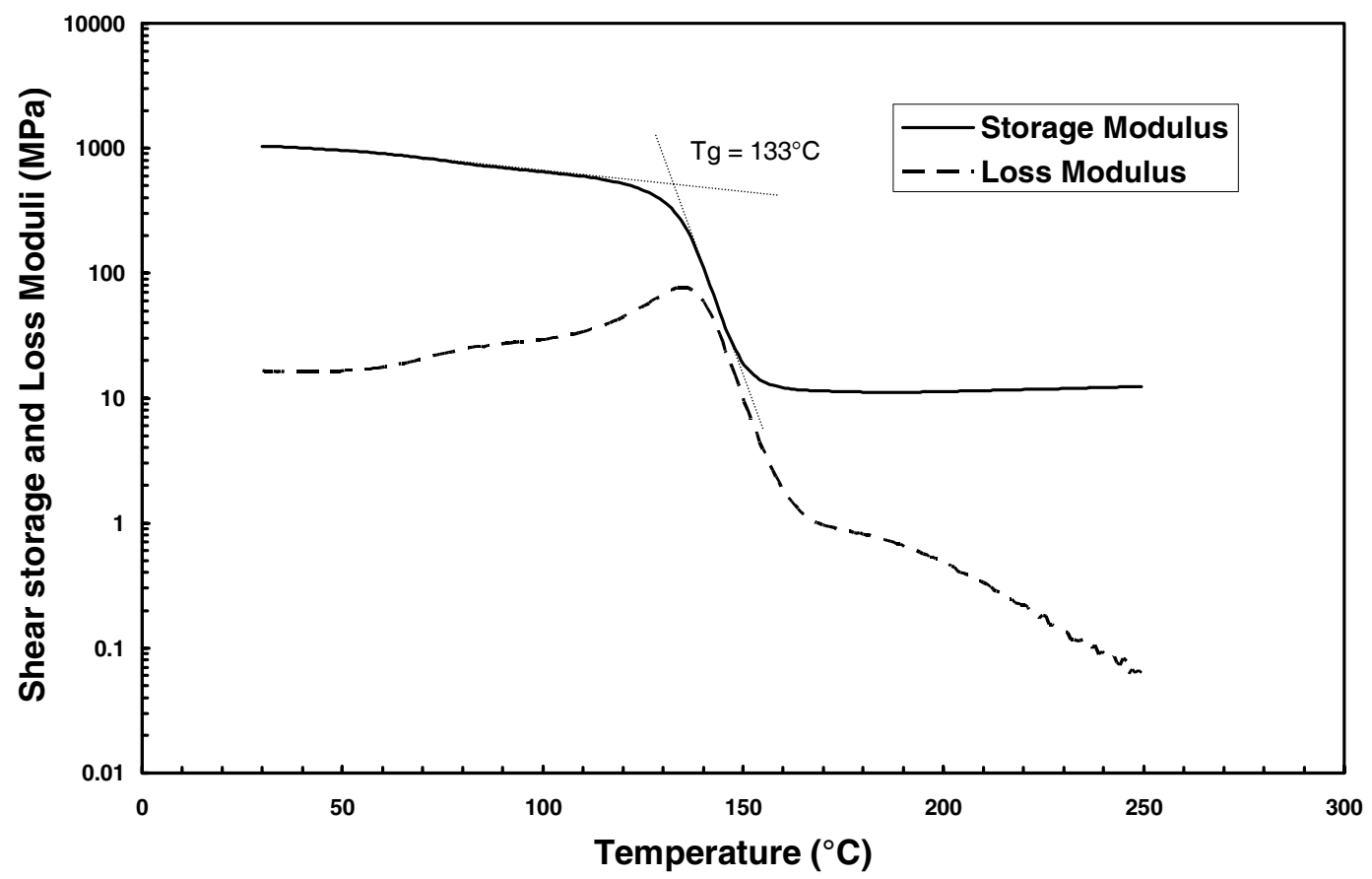

Figure 21.- Shear storage and loss moduli for E-862 resin.

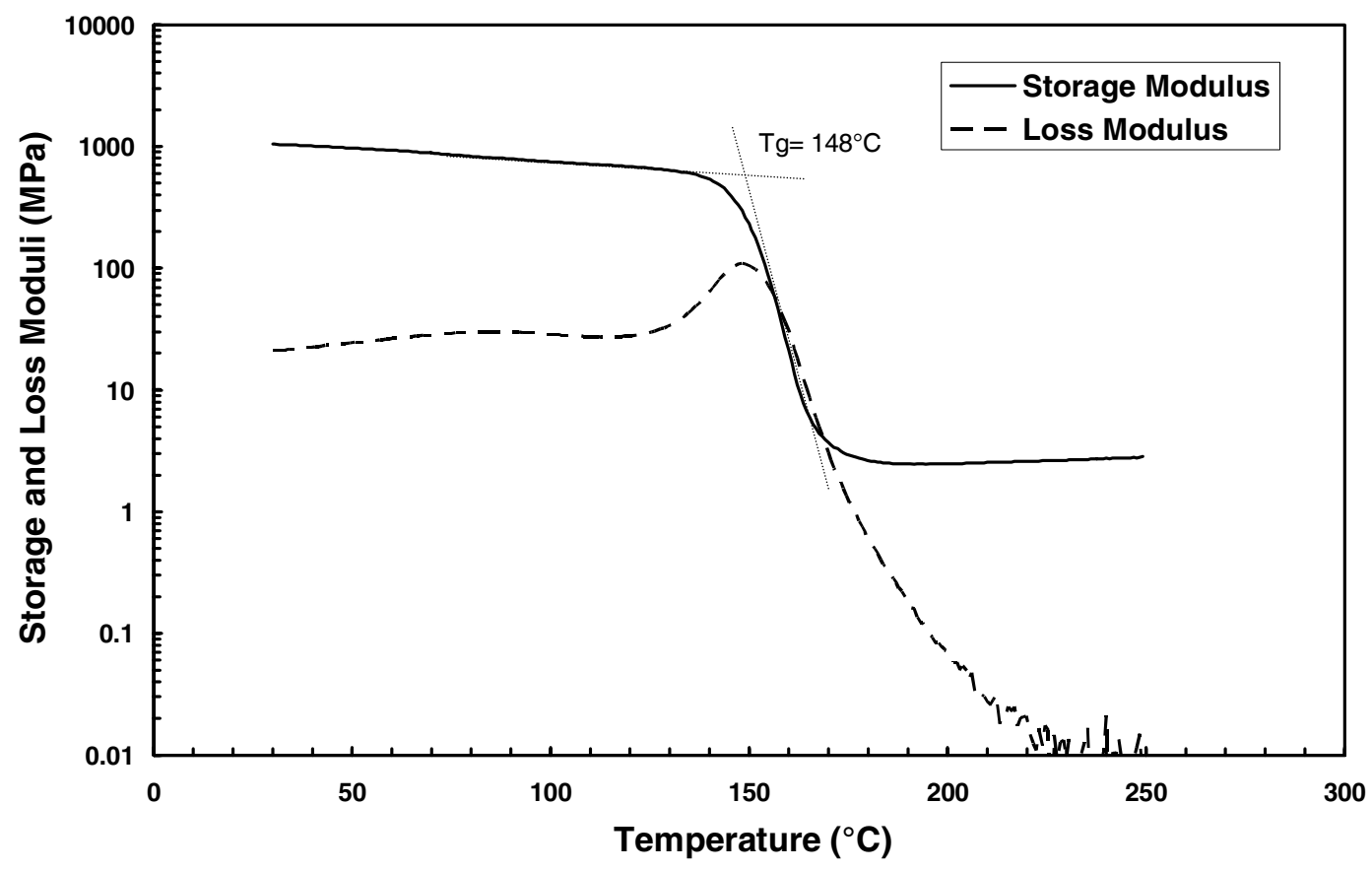

Figure 22.- Shear storage and loss moduli for PR-520 resin. 


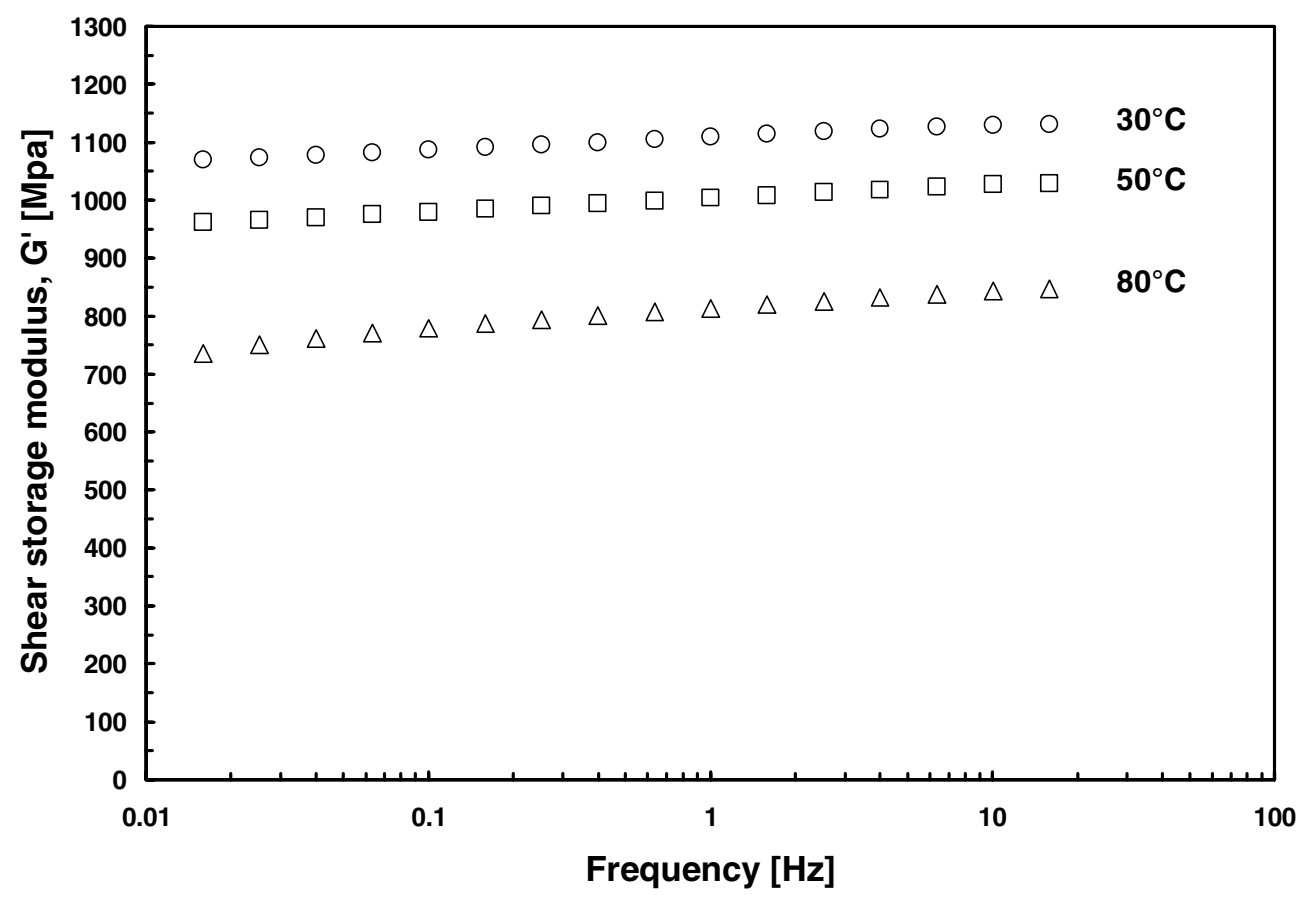

Figure 23.-Effect of test rate and temperature on the shear storage modulus for E-862 resin.

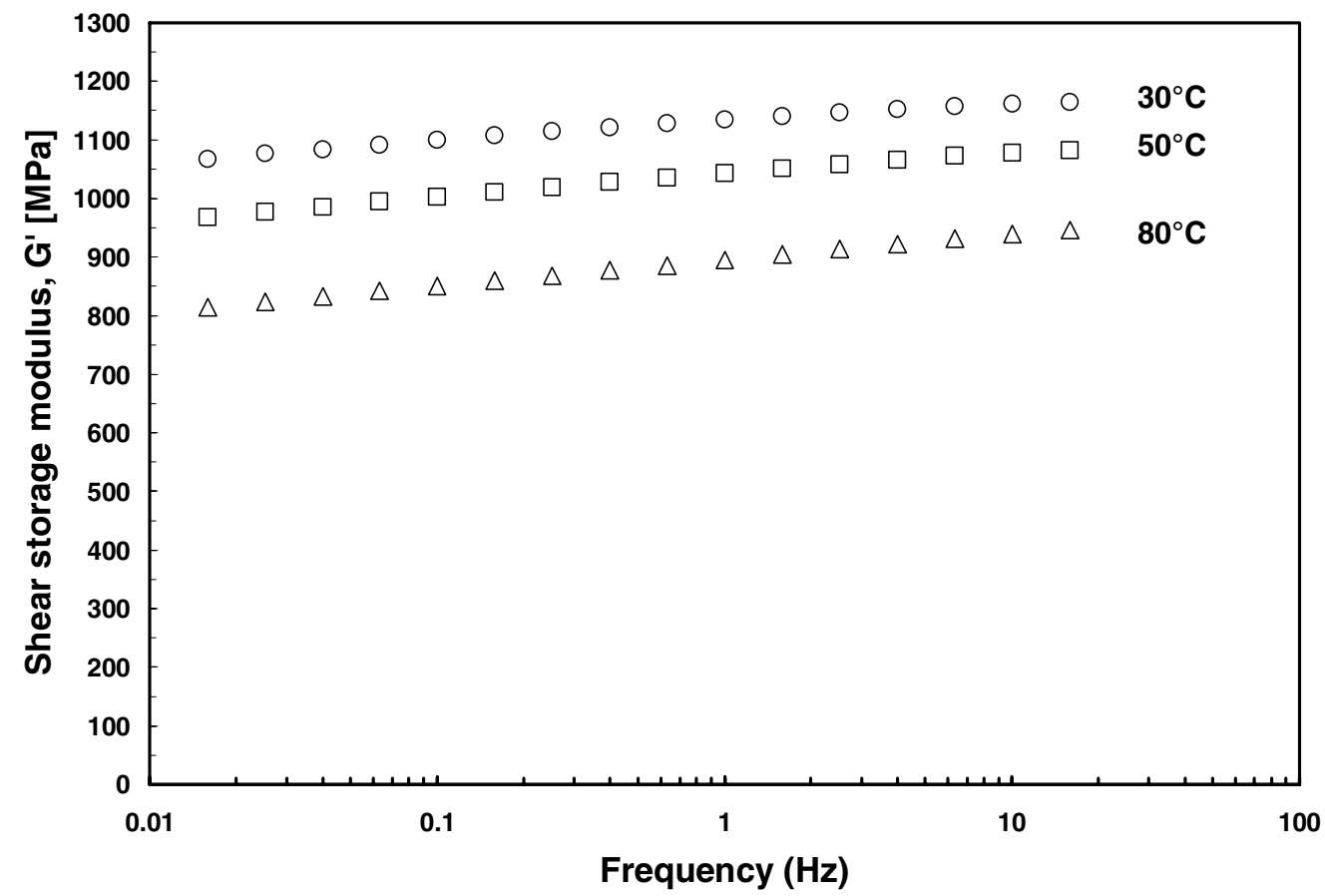

Figure 24.-Effect of test rate and temperature on the shear storage modulus for PR-520 resin. 


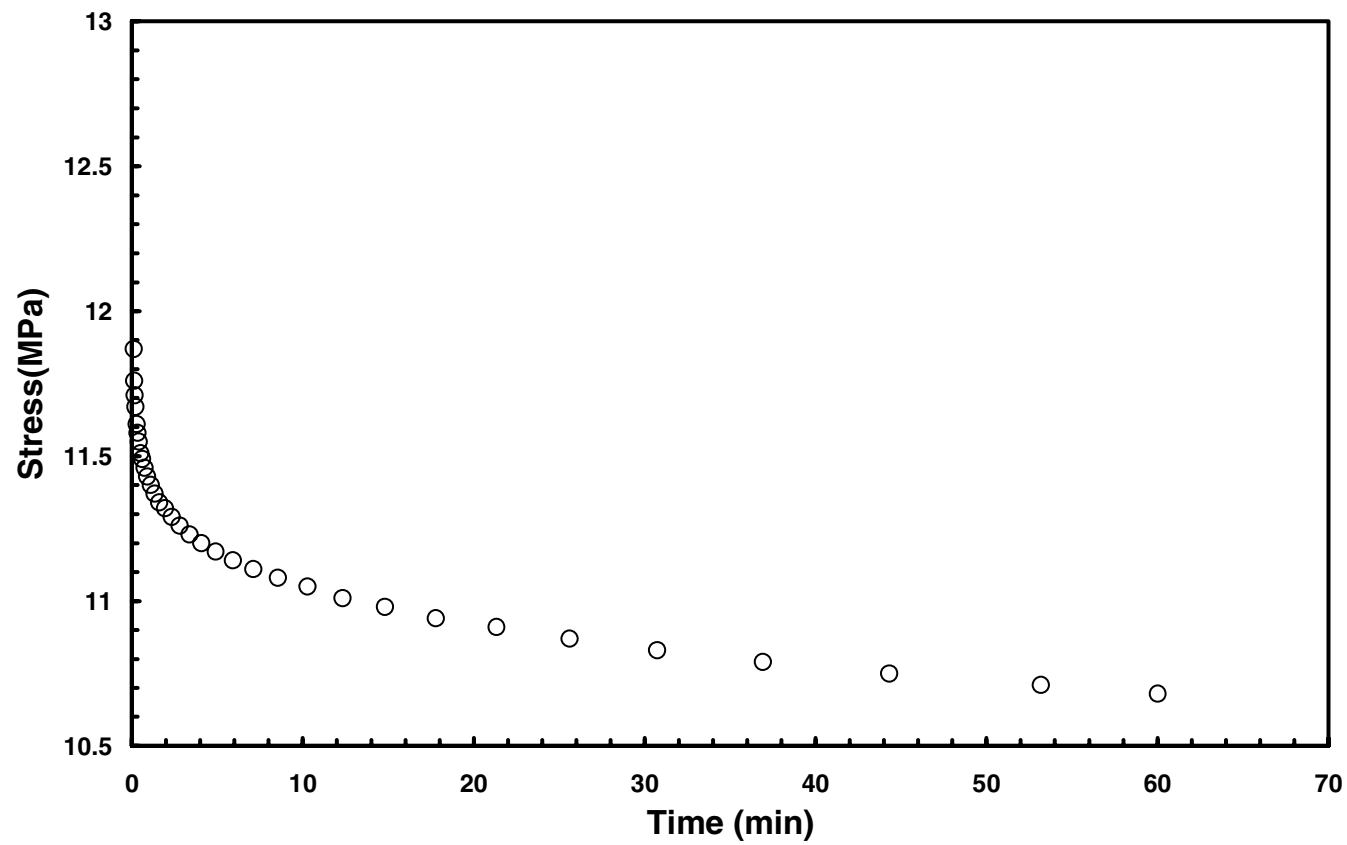

Figure 25.- Stress relaxation at $30{ }^{\circ} \mathrm{C}$ for E-862 resin.

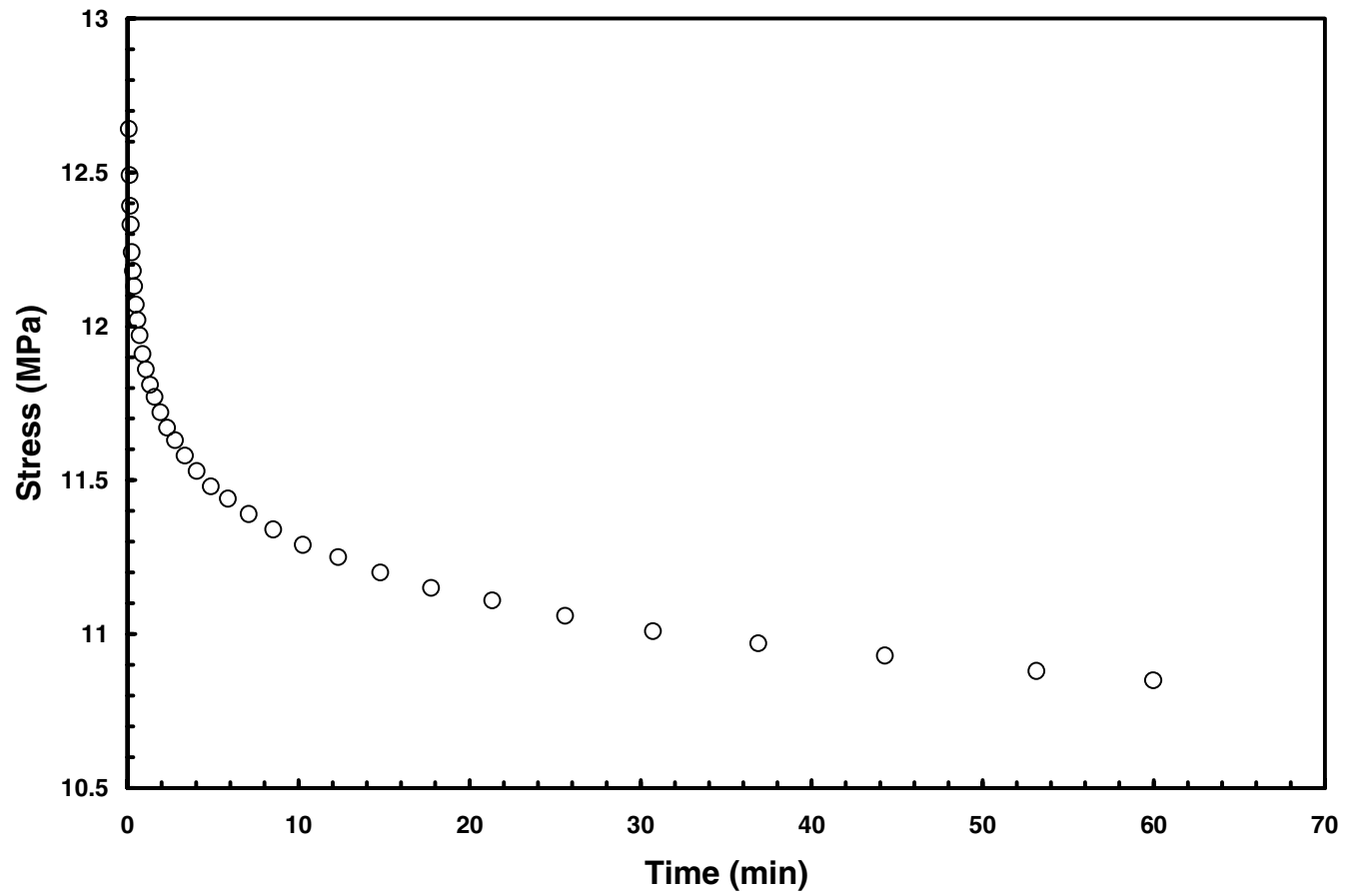

Figure 26.- Stress relaxation at $30{ }^{\circ} \mathrm{C}$ for PR-520 resin. 


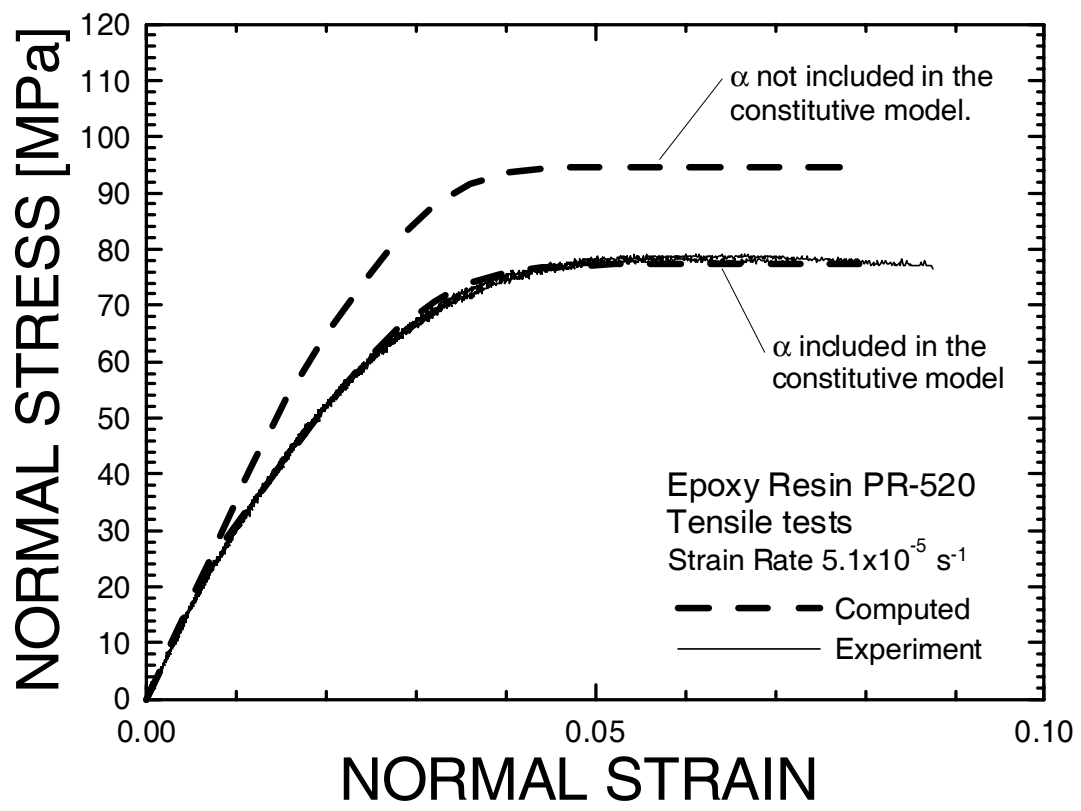

Figure 27.-The effect of the hydrostatic stress state variable $\alpha$ on the calculated stress-shear strain curves for PR-520 resin at strain rate of $5.1 \times 10^{-5} \mathrm{~s}^{-1}$ (Low Rate). 


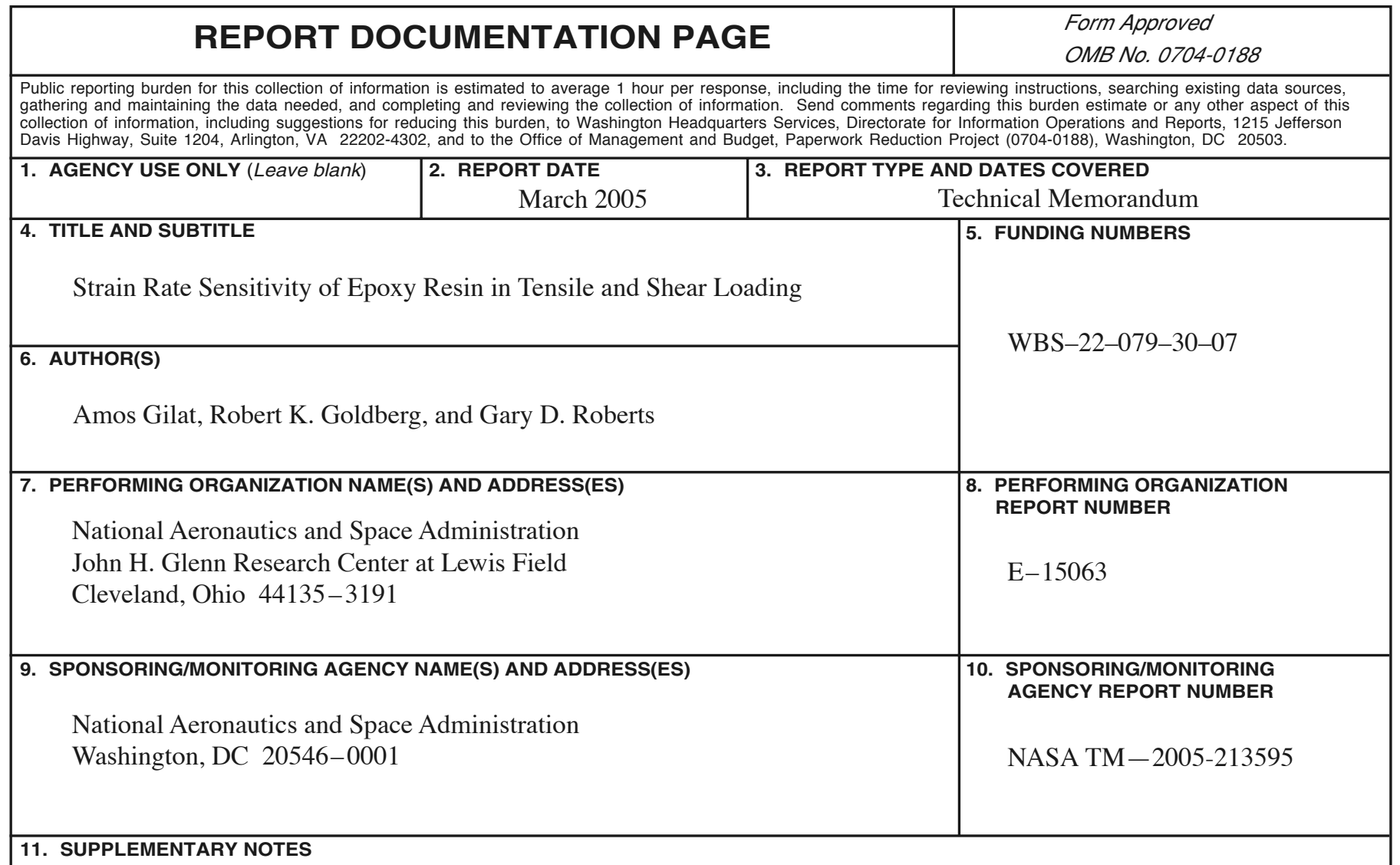

Amos Gilat, Ohio State University, Department of Mechanical Engineering, 206 West 18th Avenue, Columbus, Ohio 43210, e-mail: gilat.1@osu.edu; and Robert K. Goldberg and Gary D. Roberts, NASA Glenn Research Center. Responsible person, Gary D. Roberts, organization code RMP, 216-433-3244.

12a. DISTRIBUTION/AVAILABILITY STATEMENT

12b. DISTRIBUTION CODE

Unclassified - Unlimited

Subject Category: 27

Available electronically at http://gltrs.grc.nasa.gov

This publication is available from the NASA Center for AeroSpace Information, 301-621-0390.

13. ABSTRACT (Maximum 200 words)

The mechanical response of E-862 and PR-520 resins is investigated in tensile and shear loadings. At both types of loading the resins are tested at strain rates of about $5 \times 10^{-5}, 2$, and 450 to $700 \mathrm{~s}^{-1}$. In addition, dynamic shear modulus tests are carried out at various frequencies and temperatures, and tensile stress relaxation tests are conducted at room temperature. The results show that the toughened PR-520 resin can carry higher stresses than the untoughened E-862 resin. Strain rate has a significant effect on the response of both resins. In shear, both resins show a ductile response with maximum stress that is increasing with strain rate. In tension a ductile response is observed at low strain rate $\left(\sim 5 \times 10^{-5} \mathrm{~s}^{-1}\right)$, and brittle response is observed at the medium and high strain rates $\left(2\right.$, and $\left.700 \mathrm{~s}^{-1}\right)$. The hydrostatic component of the stress in the tensile tests causes premature failure in the E-862 resin. Localized deformation develops in the PR-520 resin when loaded in shear. An internal state variable constitutive model is proposed for modeling the response of the resins. The model includes a state variable that accounts for the effect of the hydrostatic component of the stress on the deformation.

14. SUBJECT TERMS

Polymer matrix composites; Impact; Strain rate; Polymers; Epoxy; Resin

\begin{tabular}{|c|c|c|}
\hline $\begin{array}{c}\text { 17. SECURITY CLASSIFICATION } \\
\text { OF REPORT } \\
\text { Unclassified }\end{array}$ & $\begin{array}{c}\text { 18. SECURITY CLASSIFICATION } \\
\text { OF THIS PAGE } \\
\text { Unclassified }\end{array}$ & $\begin{array}{c}\text { 19. SECURITY CLASSIFICATION } \\
\text { OF ABSTRACT } \\
\text { Unclassified }\end{array}$ \\
\hline
\end{tabular}

NSN 7540-01-280-5500

Standard Form 298 (Rev. 2-89)

Prescribed by ANSI Std. Z39-18 298-102 

